\title{
Interspike Intervals Reveal Functionally Distinct Cell Populations in the Medial Entorhinal Cortex
}

\author{
Patrick Latuske, Oana Toader, and Kevin Allen \\ Department of Clinical Neurobiology, Medical Faculty of Heidelberg University and German Cancer Research Center (Deutsches Krebsforschungszentrum), \\ 69120 Heidelberg, Germany
}

The superficial layers of the medial entorhinal cortex (MEC) contain spatially selective neurons that are crucial for spatial navigation and memory. These highly specialized neurons include grid cells, border cells, head-direction cells, and irregular spatially selective cells. In addition, MEC neurons display a large variability in their spike patterns at a millisecond time scale. In this study, we analyzed spike trains of neurons in the MEC superficial layers of mice and found that these neurons can be classified into two groups based on their propensity to fire spike doublets at 125-250 Hz. The two groups, labeled "bursty" and "non-bursty" neurons, differed in their spike waveforms and interspike interval adaptation but displayed a similar mean firing rate. Grid cell spatial periodicity was more commonly observed in bursty than in non-bursty neurons. In contrast, most neurons with head-direction selectivity or those that fired at the border of the environment were non-bursty neurons. During theta oscillations, both bursty and non-bursty neurons fired preferentially near the end of the descending phase of the cycle, but the spikes of bursty neurons occurred at an earlier phase than those of non-bursty neurons. Finally, analysis of spike-time crosscorrelations between simultaneously recorded neurons suggested that the two cell classes are differentially coupled to fast-spiking interneurons: bursty neurons were twice as likely to have excitatory interactions with putative interneurons as non-bursty neurons. These results demonstrate that bursty and non-bursty neurons are differentially integrated in the MEC network and preferentially encode distinct spatial signals.

Key words: border cells; burst; entorhinal cortex; grid cells; head-direction cells

\section{Significance Statement}

We report that neurons in the superficial layers of the medial entorhinal cortex can be classified based on their tendency to fire bursts of action potentials at $125-250 \mathrm{~Hz}$. The relevance of this classification is demonstrated by the types of spatial information preferentially encoded by bursty and non-bursty neurons. Grid-like spatial periodicity is more commonly observed in bursty neurons, whereas most cells with head-direction selectivity or those that are firing at the border of the environment are non-bursty neurons. This work indicates that the spatial firing patterns of neurons in the medial entorhinal cortex can be predicted by electrophysiological features reflecting the synaptic inputs and/or integrating properties of the neurons.

\section{Introduction}

The hippocampus and parahippocampal area are essential for the rapid encoding of memory with strong temporal and spatial content (Morris et al., 1982; Bannerman et al., 2001; Day et al., 2003; MacDonald et al., 2011). The spatial aspect of hippocampus-dependent memory relies on spatially selective neurons in the medial entorhinal

\footnotetext{
Received Jan. 21, 2015; revised June 5, 2015; accepted June 29, 2015.

Author contributions: P.L. and K.A. designed research; P.L., O.T., and K.A. performed research; P.L., O.T., and K.A. analyzed data; P.L. and K.A. wrote the paper.

This work was supported by Emmy Noether Programme Grant AL 1730/1-1 to K.A. and Collaborative Research Centre Grant SFB-1134 from the Deutsche Forschungsgemeinschaft.

The authors declare no competing financial interests.

Correspondence should be addressed to Dr. Kevin Allen, Department of Clinical Neurobiology, DKFZ/A230, Im Neuenheimer Feld 280,69120 Heidelberg, Germany. E-mail: allen@uni-heidelberg.de.

DOI:10.1523/JNEUROSCI.0276-15.2015

Copyright $\odot 2015$ the authors $\quad 0270-6474 / 15 / 3510963-14 \$ 15.00 / 0$
}

cortex (MEC) that generate a metric representation of space that has grid cells as one of its principal units (Hafting et al., 2005; Steffenach et al., 2005; McNaughton et al., 2006; Sargolini et al., 2006). Besides grid cells, the MEC comprises several other functionally defined cell types, including head-direction cells, border cells, grid $\times$ headdirection conjunctive cells, and irregular spatially selective cells (Sargolini et al., 2006; Fyhn et al., 2008; Savelli et al., 2008; Solstad et al., 2008). These functional cell types are all found in superficial layers II and III of the MEC, which provides the majority of the spatially modulated firing inputs received by the hippocampus (Hargreaves et al., 2005; Sargolini et al., 2006; Zhang et al., 2013).

A key step in understanding the neuronal network in the superficial layers of the MEC is to determine the anatomical, molecular, and electrophysiological features with which MEC neurons can be classified. Juxtacellular and whole-cell recordings, both allowing an- 
atomical identification of the recorded neurons, have been used to contrast the firing patterns of two of the principal anatomical cell types of layer II, namely the pyramidal and stellate cells (Varga et al., 2010; Domnisoru et al., 2013; Schmidt-Hieber and Häusser, 2013; Ray et al., 2014). Although it appears that the firing of both pyramidal and stellate cells displays grid cell spatial periodicity (Domnisoru et al., 2013), the preponderance of the grid pattern in the two anatomical cell types and their respective firing properties during theta oscillations are still debated (Schmidt-Hieber and Häusser, 2013; Tang et al., 2014). Besides these two excitatory cell types, the firing pattern of parvalbumin-expressing interneurons, which represent $\sim 50 \%$ of the GABAergic neurons in the superficial layers of the MEC, has also been characterized. In this abundant GABAergic cell type, very few cells appear to display grid cell periodicity (Buetfering et al., 2014).

An approach complementary to defining neuronal classes based on anatomical properties or molecular markers is to use electrophysiological features that are likely to reflect the synaptic inputs and integrating properties of the neurons at a millisecond time scale. This approach can be pursued with extracellular techniques, making it possible to investigate the variability in large databases that are currently not available with juxtacellular or intracellular techniques. In this study, we tested whether interspike intervals of MEC neurons can be used to identify cell populations with unique functional properties. We found that the propensity of firing spike doublets within 4-8 ms reveals two classes of MEC neurons with distinct spatial and temporal firing patterns.

\section{Materials and Methods}

Microdrive implantation. Lightweight microdrives carrying four to eight movable tetrodes were implanted above the MEC of 22 wild-type (C57BL/6) male mice. Tetrodes were made of $12-\mu \mathrm{m}$-diameter tungsten wires (H-Formvar insulation with Butyral bond coat; California Fine Wire Company) and were gold plated to reduce impedance to 300-400 $\mathrm{k} \Omega$. A small craniotomy was made above the MEC $(0.2 \mathrm{~mm}$ anterior to the transverse sinus and $3.1 \mathrm{~mm}$ lateral to bregma), and the tetrodes were lowered $0.8 \mathrm{~mm}$ into the brain with a $6^{\circ}$ angle in the posterior direction. Two stainless steel screws were inserted into the skull above the cerebellum and served as reference and ground signals. The microdrive was attached to the skull with dental cement.

Behavioral training. After a 1 week recovery period, mice were maintained at $85 \%$ of their free-feeding weight and trained to forage in a gray open field $(70 \times 70 \times 30 \mathrm{~cm}$ high $)$ made of wood. A 21 -cm-wide white cue card was attached to one wall of the open field. Food pellets (AIN-76A rodent tablets $5 \mathrm{mg}$; TestDiet) were delivered at random locations (ENV-203-5; MED Associates) to ensure that the mouse visited the entire surface of the recording environment. Recording started only once the mouse explored most of the open field within a 20 min trial.

Data acquisition. Tetrodes were lowered into the superficial layers of the MEC over several days. Large theta oscillations, a strong theta modulation of action potentials, and the occasional presence of grid cells were indicative of correct placement of the tetrodes. Tetrode location was subsequently confirmed by histological analysis. After each recording session, the tetrodes were moved down by $25-50 \mu \mathrm{m}$.

The local field potentials were passed through a unity-gain buffer preamplifier (Axona). Two different recording systems were used. For seven mice, the signal was amplified $(600 \times)$, bandpass filtered $(0.33 \mathrm{~Hz}$ to 12 $\mathrm{kHz}$ ), and digitized at $24 \mathrm{kHz}$ (16 bits/sample, DacqUSB, Axona). For 15 mice, the signal was amplified $(1000 \times)$, bandpass filtered $(0.1 \mathrm{~Hz}$ to 10 $\mathrm{kHz}$; Sensorium), and digitized at $20 \mathrm{kHz}$ (16 bits/sample, PCI-6259, National Instruments; http://savannah.nongnu.org/projects/kacq/). A video camera (Handycam, Sony) located above the recording environment was used to follow the position of light-emitting diodes (LEDs) attached to the headstage. For 18 mice, three LEDs of different colors were used to track the position of the mouse and its head direction. For four mice, only one infrared LED was used to locate the position of the mouse. The head direction was not available for these four mice. The sampling rate of the video was $25 \mathrm{~Hz}$.

Spike detection and sorting. Action potentials were detected by calculating the root mean square of the bandpass filtered signal $(0.8-5 \mathrm{kHz})$ within a sliding window $(0.2 \mathrm{~ms})$. Action potentials were detected whenever the signal exceeded the mean by $>5$ SDs. The spike waveforms on each of the four tetrode wires were extracted and submitted to a principal component analysis (Csicsvari et al., 1999). The first three principal components were calculated from each tetrode wire, resulting in a vector of 12 waveform parameters. Based on the waveform parameters, spike clusters were automatically generated using KlustaKwik (https://github. com/klusta-team/klustakwik). The clusters were further refined with a graphical user interface. Only clusters with a clear refractory period in their spike-time autocorrelation were used for further analysis. Spiketime autocorrelations were constructed by treating every spike in turn as a reference spike to all other spikes within a given time window. The spike count per $1 \mathrm{~ms}$ bin was normalized by the total number of spikes to obtain a firing probability.

A two-step procedure was used to exclude putative interneurons from the analysis. First, neurons with a mean firing rate $>10 \mathrm{~Hz}$ were identified and labeled as interneurons. Second, a linear classifier was used to exclude additional neurons with spike waveforms similar to those of interneurons. Spike asymmetry and spike duration after the trough of all neurons were used to train a binary linear classifier to separate the identified interneurons (mean firing rate $>10 \mathrm{~Hz}$ ) from all other recorded neurons ( $r$-project, e1071 package, svm function). Then, the classifier was applied to neurons with a mean firing rate $<10 \mathrm{~Hz}$, and cells classified as interneurons were removed from subsequent analysis.

Identification of neuron classes based on spike-time autocorrelations. The first $12 \mathrm{~ms}$ of the spike-time autocorrelation $(0.5 \mathrm{~ms} / \mathrm{bin})$ for each neuron was used in a principal component analysis to identify the components with the most variance (r-project, stats package, prcomp function). Principal components were computed and ranked by using singular value decomposition of the zero-centered data matrix. To assign cluster identity to each neuron, the $k$-means cluster algorithm was applied to the first three principal components of the spike-time autocorrelations ( $\mathrm{r}$ project, stats package, kmeans function). The number of clusters $(k=2)$ was determined visually, and the maximum number of iterations was set to 100 . As the starting conditions of the clustering algorithm are chosen randomly, the analysis was repeated 20 times with different initial configurations to obtain the best fit, which was defined as the minimal sum of squares from the points to the assigned cluster center.

Interspike interval adaptation was assessed during spike triplets, which were defined as three spikes fired within a $50 \mathrm{~ms}$ window that was not preceded by an additional spike in the previous $50 \mathrm{~ms}$. Moreover, to be considered, spike triplets had to be preceded by at least one spike 50-200 $\mathrm{ms}$ before the first spike of the triplet. The mean first and second interspike intervals of spike triplets were calculated. The ratio between the first and second interspike intervals was used as the interspike interval adaptation score.

Spatial properties of neurons. Spatial firing rate maps were generated by dividing the environment into $2 \times 2 \mathrm{~cm}$ bins. An occupancy map was defined as the time spent in each bin and was smoothed with a Gaussian kernel $(S D=3 \mathrm{~cm})$. The number of spikes emitted as the animal was in each bin was also calculated. The firing rate per bin was obtained by dividing the spike count of a bin by the corresponding bin of the occupancy map. The resulting firing rate map was smoothed with a Gaussian kernel function $(\mathrm{SD}=3 \mathrm{~cm})$. Only periods when the mouse ran faster than $3 \mathrm{~cm} / \mathrm{s}$ were considered. The spatial information score of a neuron (Skaggs et al., 1996) was defined as follows:

$$
I=\sum_{i=1}^{N} p_{i} \frac{\lambda_{i}}{\lambda} \log _{2} \frac{\lambda_{i}}{\lambda},
$$

where $p_{\mathrm{i}}$ is the occupancy probability of bin $i$ in the firing map, $\lambda_{\mathrm{i}}$ is the firing rate in bin $i$, and $\lambda$ is the mean firing rate of the neuron. A cell was considered spatially selective if its information score exceeded the 95th 
percentile $(I=0.48)$ of a distribution of information scores obtained by a shuffling procedure in which the spike times of cells were shifted by a minimum of $20 \mathrm{~s}$ before recalculating the spatial information scores. The shuffling procedure was repeated 500 times for each neuron.

Spatial autocorrelations were calculated from the firing rate maps (Hafting et al., 2005). The bins of a spatial autocorrelation matrix represented Pearson correlation coefficients between all possible pairs of bins in a firing rate map with a given spatial lag. The spatial lag associated with a particular bin of the matrix was determined by the location of that bin relative to the matrix center. Pearson correlations at spatial lags for which $<20$ pairs of firing rates were available were not considered (Hafting et al., 2005). Peaks in the autocorrelation matrix were defined as $>10$ adjacent bins with a correlation coefficient higher than a peak detection threshold set to 0.1 . The $60^{\circ}$ periodicity in the spatial autocorrelation matrix was estimated by the grid score (Sargolini et al., 2006). A circular region of the spatial autocorrelation matrix containing up to six peaks and excluding the central peak was defined. Pearson correlation coefficients $(r)$ were calculated between this circular region of the matrix and a rotated version of itself (by $30,60,90,120$, and $150^{\circ}$ ). The grid score was obtained using the following formula:

$$
\left(\frac{r 60^{\circ}+r 120^{\circ}}{2}\right)-\left(\frac{r 30^{\circ}+r 90^{\circ}+r 150^{\circ}}{3}\right) .
$$

The probability of obtaining a given grid score by chance was estimated by shifting the spike times of the cells by a minimum of $20 \mathrm{~s}$ before recalculating the firing rate map and the grid score. This procedure was repeated 500 times for each neuron. Neurons with a grid score associated with a probability of $<1 \%(0.54)$ were considered grid cells.

Head-direction cells were identified by generating a histogram with the firing rate of a cell as a function of head direction $\left(10^{\circ} / \mathrm{bin}\right.$; smoothing kernel $\mathrm{SD}=15^{\circ}$ ). A mean vector length was calculated from this histogram. Neurons with a vector length $>0.4$ were considered headdirection cells.

A border score adapted from Solstad et al. (2008) was calculated for each neuron to identify border cells. The pixels of a firing rate map that were directly adjacent to each wall of the open field were identified. Firing fields, defined as groups of adjacent pixels with a firing rate larger than $30 \%$ of the peak firing rate of the map and covering at least $80 \mathrm{~cm}^{2}$, were detected. For each field, the proportion of the pixels along a wall that were also part of the field was calculated for the four walls separately. CM was defined as the maximum proportion obtained over all possible field-wall combinations. DM was the mean shortest distance to a wall for pixels that were part of a firing field, weighted by the firing rate in each pixel. DM was then normalized so that it ranged from 0 to 1 . For each pixel in the map, the shortest distance to a wall was calculated. The largest value obtained over all map pixels was the value used for the normalization of DM. The border score was obtained with the following formula:

$$
\frac{C M-D M}{C M+D M} \text {. }
$$

A score of -1.0 was obtained if a neuron had only firing fields that did not touch any of the walls. Neurons with no firing field $(n=5)$ were not considered in the analysis of the border score. Border cells were defined as cells with a border score above 0.528 .

Neurons with a significant spatial information score $(>0.48)$ that did not reach the criteria of other spatially defined cell types (grid score $<0.54$; head-direction vector length $<0.4$; border score $<0.528$ ) were defined as irregular spatially selective neurons. Neurons with a nonsignificant spatial information score, grid score, and head-direction vector length were considered nonspatially selective neurons (spatial information score $<0.48$; grid score $<0.54$; head-direction vector length $<0.4$; border score $<0.528$ ).

Theta oscillations. Theta oscillations were detected on each tetrode. The raw signal was bandpass filtered at delta $(2-4 \mathrm{~Hz})$ and theta $(6-10$ $\mathrm{Hz}$ ) frequencies. The power (root mean square) in $500 \mathrm{~ms}$ windows was calculated for the two frequency bands. Epochs with a theta/delta power ratio $>2$ were defined as theta epochs. To detect individual theta cycles, the raw signal was bandpass filtered $(5-14 \mathrm{~Hz})$, and the positive-to- negative zero crossings were detected within theta epochs. Phase $0^{\circ}$ was defined as the positive-to-negative zero crossing of the oscillation. The theta phase of spikes was obtained by linear interpolation, and the preferred theta phase of a neuron was defined as the circular mean of its spikes.

The intrinsic theta frequency of a cell was calculated from its instantaneous firing rate. The number of spikes in $1 \mathrm{~ms}$ time windows was counted. The convolution between the spike count array and a Gaussian kernel $(\mathrm{SD}=5 \mathrm{~ms}$ ) was performed. The resulting vector was integrated over $5 \mathrm{~ms}$ time windows, and the values were multiplied by 1000/5 to transform the probability into hertz. The power spectrum of the instantaneous firing rate was calculated, and a theta rhythmicity index was defined as follows:

$$
\frac{\theta-\text { baseline }}{\theta+\text { baseline }},
$$

where $\theta$ is the mean power at theta frequency $(6-10 \mathrm{~Hz})$ and baseline is the mean power in two adjacent frequency bands $(3-5$ and $11-13 \mathrm{~Hz}$ ). The statistical significance of theta rhythmicity was estimated by adding a random jitter (from -65 to $65 \mathrm{~ms}$, uniform distribution) to the spike times and recalculating theta rhythmicity indices. The 95th percentile (0.032) of the surrogate indices served as the significance threshold. The intrinsic theta frequency of neurons, defined as the frequency between 6 and $10 \mathrm{~Hz}$ with the largest power, was calculated for neurons with significant theta rhythmicity.

Classification of neurons into putative calbindin ${ }^{+}$and calbindin ${ }^{-}$neurons. Classification of neurons as putative calbindin ${ }^{+}$and calbindin ${ }^{-}$ cells was performed as described previously (Tang et al., 2014). The preferred theta phase $(\psi)$ and theta vector length $(S)$ of identified calbin$\operatorname{din}^{+}$and calbindin ${ }^{-}$cells were taken from the work of Tang et al. (2014). The classification was performed in the Euclidean space with the vectors $(\cos (\psi) S, \sin (\psi) S)$. The vectors were used to train a support vector machine with a Gaussian radial basis function kernel (scikit-learn 0.16.1 module, Python 2.7.5). We used the classifier to predict the putative anatomical cell types of bursty and non-bursty neurons. Cells with a distance $<0.1$ from the decision hyperplane were removed for better separation of the cell groups.

Crosscorrelation analysis and putative excitatory connections. Putative excitatory connections were detected as narrow peaks in the spike-time crosscorrelation of simultaneously recorded neurons (from -50 to 50 ms; bin size, 0.5 ms; Csicsvari et al., 1998; Marshall et al., 2002; Maurer et al., 2006; Mizuseki et al., 2009; Dupret et al., 2013). A cross-correlogram was calculated for all pairs of neurons recorded simultaneously. For a given pair of neurons $A$ and $B$, where neuron $A$ is the reference neuron, the spikes of neuron $A$ served in turn as a reference spike. The spikes of neuron $B$ falling within $50 \mathrm{~ms}$ of a reference spike were added to the cross-correlogram in the bin corresponding to the time difference between the spike of neuron $B$ and the reference spike. Only crosscorrelograms containing at least 300 spikes of neuron $B$ were considered. The spike counts in the crosscorrelograms were then divided by the number of reference spikes to obtain a probability of observing a spike of neuron $B$ in each bin. The bins from -50 to $0 \mathrm{~ms}$ and from 10 to $50 \mathrm{~ms}$ served to calculate a baseline mean and SD. A peak at short latency in the crosscorrelation was defined as at least one bin between 1 and $4 \mathrm{~ms}$ that was above 5 SDs from the baseline mean. To exclude pairs for which the baseline was not stable (e.g., a crosscorrelation with the peak beginning before time 0 ), any crosscorrelation in which a bin between -10 and $0 \mathrm{~ms}$ or between 10 and $50 \mathrm{~ms}$ exceeded 3 SDs from the baseline mean was excluded from the analysis.

\section{Results}

We recorded cell activity $(n=1236)$ and local field potentials in the superficial layers of the MEC in 22 mice as they explored a $70 \times 70 \mathrm{~cm}$ square box (Fig. $1 A, B$ ). Histological examination confirmed that the majority of the tetrodes were located in the most dorsal region of the MEC, although a small fraction of the tetrodes $(\sim 15 \%)$ were found in the parasubiculum, close to 
the border between the parasubiculum and the MEC. Tetrode tips went from deeper to superficial layers, with a median angle of $25.78^{\circ}$ between the tetrode track and the MEC pia surface $\left(\mathrm{Q} 1=20.28^{\circ}\right.$, Q3 $=29.86^{\circ}$ ). Most tetrode tips had reached layer II by the end of the experiment. A total of $37.9 \%$ of the neurons (468 of 1236) had a significant spatial information score, and $15.4 \%$ were classified as grid cells (Fig. 1B).

Qualitative inspection of the spiketime autocorrelations of the neurons revealed a surprisingly large variability (Fig. $1 B, C)$. Whereas some autocorrelations had a well defined peak within $4-8 \mathrm{~ms}$, others only showed a peak at a later time point (Fig. 1C, right). We therefore tested whether principal neurons of the MEC could be classified based on their spike patterns within $12 \mathrm{~ms}$ time windows. $\mathrm{Pu}-$ tative interneurons were excluded from the analysis (putative interneurons, $n=$ 318; principal neurons, $n=918$ ).

\section{Identification of bursty and non-bursty neurons}

We performed a principal component analysis on the first $12 \mathrm{~ms}$ of the spiketime autocorrelations $(0.5 \mathrm{~ms} / \mathrm{bin}$, peak normalized to 1 ). When the first two principal components were plotted against each other, the neurons fell on a C-shaped structure with two principal axes (Fig. $2 A)$. The first three principal components accounted for $84.35 \%$ of the variance in the data $(\mathrm{PC} 1=52.60 \%, \mathrm{PC} 2=25.87 \%$, PC3 $=5.88 \%)$. A $k$-means cluster algorithm $(k=2)$ was used to assign class membership to neurons (Fig. 2B). A linear discriminant analysis was then applied to find the linear combination of the bins of the autocorrelation that best separated the two groups. The resulting distribution exhibited two clear modes with a small overlap between the two groups (Fig. 2C). The spike-time autocorrelations in the two groups had different shapes, and the main difference was the tendency to fire spike doublets within $4-8 \mathrm{~ms}$ (Fig. $2 B-D$ ). In the remainder of this manuscript, the neurons of the two groups are labeled "bursty" and "non-bursty." Neurons falling in the region of the $x$-axis where the two groups overlapped were removed from subsequent analysis (Fig. 2C, right; 102 of 918 neurons were excluded; bursty, $n=410$; non-bursty, $n=406$ ). This exclusion allowed a clearer comparison of the two groups but did not affect the conclusions of the study. Using longer spike-time autocorrelations (e.g., 0-30 ms) led to a similar assignation of the neurons, with only $4 \%$ of the neurons changing groups.

Figure $2 E$ shows the cumulative distribution of interspike intervals for bursty and non-bursty neurons. Compared with nonbursty neurons, bursty neurons had more interspike intervals
B
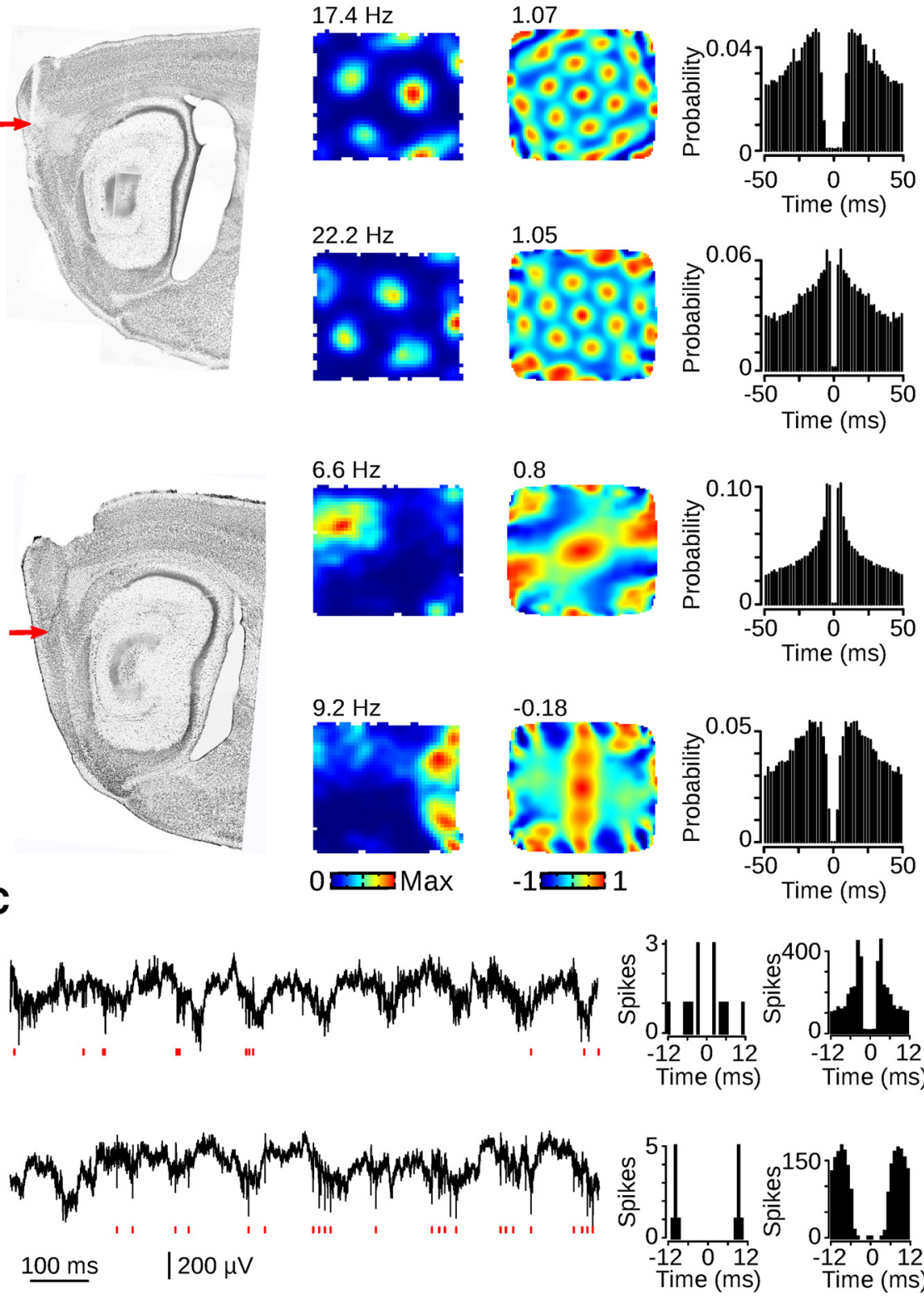

Figure 1. Variability in spike-time autocorrelations of neurons recorded in the superficial layers of the MEC. $A$, Sagittal brain sections stained with cresyl violet showing tetrode tracks reaching the superficial layers of the MEC. Tetrode tips are indicated by red arrows. $\boldsymbol{B}$, Firing rate maps, spatial autocorrelations, and spike-time autocorrelations of four neurons. Note the variability in the shape of the spike-time autocorrelations. Peak firing rates and grid scores are displayed above the firing rate maps and spatial autocorrelations, respectively. $C$, Left, Unfiltered local field potentials of two $1 \mathrm{~s}$ time windows recorded on the same tetrode. The spikes of two neurons are depicted as red lines below the field potentials. Right, Spike-time autocorrelations of the two neurons. The autocorrelations on the left contain the spikes of the $1 \mathrm{~s}$ traces, whereas those on the right are for spikes of the entire recording session. Only the neuron on the top trace fired bursts of spikes with interspike intervals of $\sim 4 \mathrm{~ms}$.

shorter than $20 \mathrm{~ms}$. We also established the minimal recording time that allowed accurate classification of neurons by the linear discriminant analysis. The classification procedure was repeated for all principal neurons, using the entire recording session but leaving a 12 min period out to test classification accuracy. When all principal neurons were included, the probability of correctly classifying a neuron as bursty or non-bursty reached $80 \%$ within $9 \mathrm{~min}$ (Fig. $2 \mathrm{~F}$ ). With a 0.3 exclusion zone around the hyperplane separating bursty and non-bursty neurons, $80 \%$ accuracy was reached within $7 \mathrm{~min}$, and only $8-15 \%$ of the neurons had to be excluded. This demonstrates that the bursty or non-bursty iden- 
A

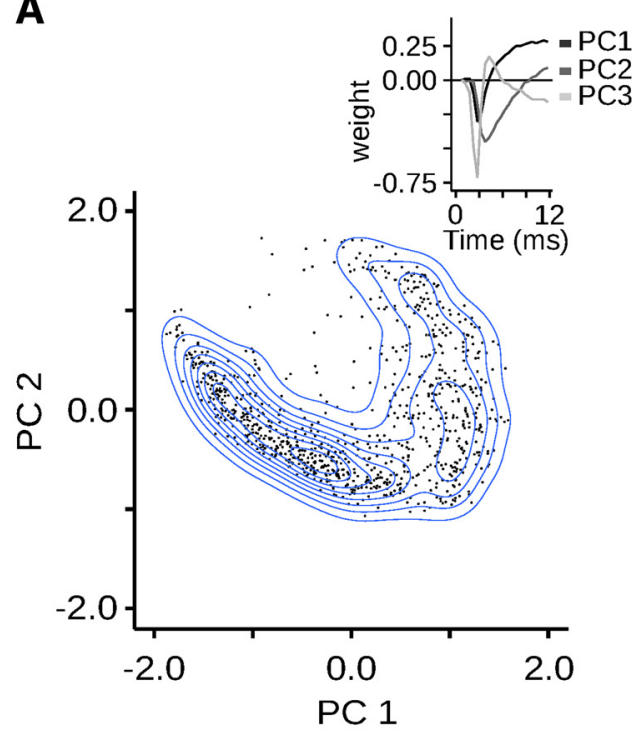

B

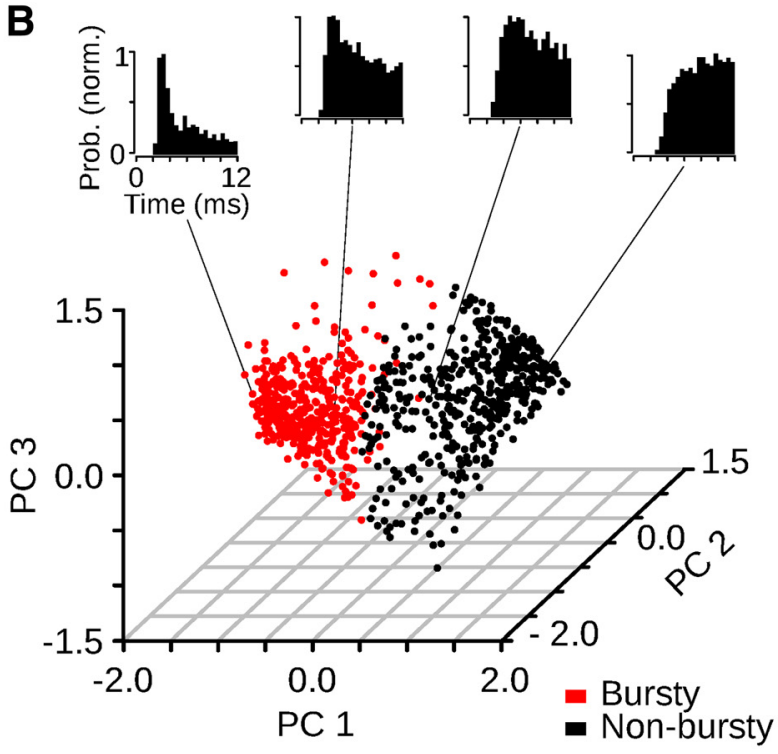

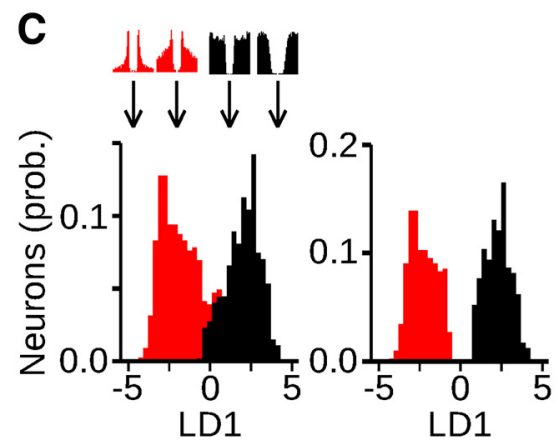
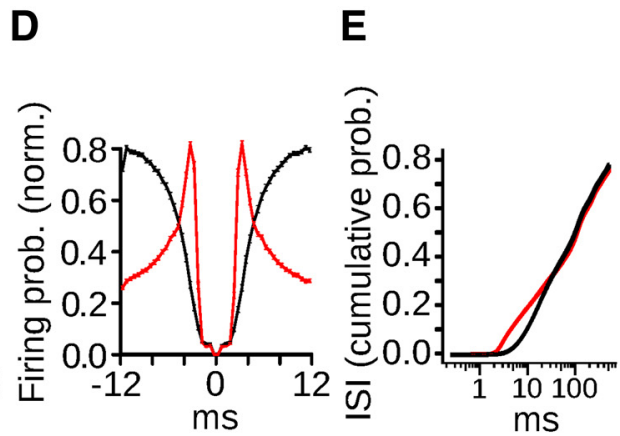

$\mathbf{F}$

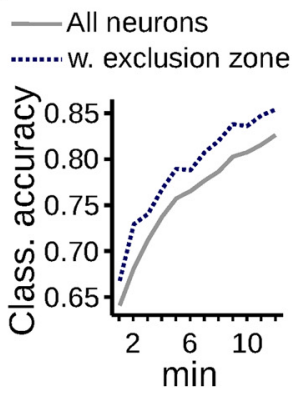

Figure 2. Identification of bursty and non-bursty neurons from spike-time autocorrelations. A, A principal component analysis was performed on the first $12 \mathrm{~ms}$ of the spike-time autocorrelations. The scatter plot shows the first two principal components. Neurons form a C-shaped structure with two main axes. A 2D kernel smoothed density estimate indicates centers of mass. Inset, Weights of the first three principal components for the autocorrelation time vector. $B, 3 D$ scatter plot of the first three principal components with $k$-means assignment of class membership $(k=2)$. Red, Bursty neurons; black, non-bursty neurons. Above the scatter plot are four examples of spike-time autocorrelations, ranging from bursty (left) to non-bursty (right). C, Left, Linear discriminant analysis of the spike-time autocorrelations to separate bursty and non-bursty neurons. All principal neurons are shown. Inset, Examples of spike-time autocorrelations of neurons with different scores. Right, Same as on the left but without the "intermediate" neurons falling in the region of the $x$-axis where bursty and non-bursty neurons overlap. D, Mean spike-time autocorrelation of bursty and non-bursty neurons. $\boldsymbol{E}$, Cumulative distribution of interspike intervals. Bursty neurons emitted more spikes with interspike intervals shorter than $20 \mathrm{~ms}$. $F$, Classification accuracy of bursty and non-bursty neurons when shorter recording periods are considered to calculate the spike-time autocorrelations. The gray line indicates classification accuracy when all principal neurons are included. The dotted blue line indicates accuracy if a 0.3 exclusion zone is present on both sides of the hyperplane separating bursty and non-bursty neurons.

tity of neurons can be established with a high degree of certainty within a 10 min recording session.

We assessed whether the spike clusters of bursty and nonbursty neurons were well isolated from the spikes of other neurons recorded simultaneously on the same tetrode. The isolation distance of each neuron was calculated (Schmitzer-Torbert et al., 2005). Spike cluster separation was slightly better for non-bursty than for bursty neurons (median: bursty, 20.04; non-bursty, 23.49; Wilcoxon rank sum test, $W=96,809.5, p<10^{-4}$ ).

Spike waveforms, interspike interval adaptation, and anatomical clustering of bursty and non-bursty neurons To test whether bursty and non-bursty neurons differed in their intrinsic biophysical properties, we compared the spike waveforms in the two groups (Fig. $3 A$ ). To prevent spike bursts from affecting this analysis, only spikes that were not preceded by another spike from the same neuron within $250 \mathrm{~ms}$ were used. We found that spike duration was longer for bursty neurons than for non-bursty neurons (Fig. 3B; mean \pm SEM: bursty, $0.327 \pm$ $0.003 \mathrm{~ms}$; non-bursty, $0.285 \pm 0.004 \mathrm{~ms}$; Wilcoxon rank sum test,
$\left.W=114,429, p<10^{-15}\right)$. Moreover, spike asymmetry tended to be larger for non-bursty than for bursty neurons (Fig. 3C; bursty, $-0.035 \pm 0.014$; non-bursty, $0.174 \pm 0.020 ; W=57,418, p<$ $\left.10^{-13}\right)$. The differences in spike waveform suggest that the neurons in the two groups differ in their morphology and/or action potential kinetics.

There was no difference in mean firing rate between bursty and non-bursty neurons (Fig. 3D; median: bursty, $1.76 \mathrm{~Hz}$; nonbursty, $1.64 \mathrm{~Hz}$; Wilcoxon rank sum test, $W=81,668, p=0.64$ ). However, the two groups differed in how their interspike intervals changed during periods of high firing activity. We detected spike triplets (three spikes within $50 \mathrm{~ms}$ ) and calculated the mean first and second interspike intervals (Fig. 3E). As expected, bursty neurons had shorter interspike intervals than non-bursty neurons. To quantify interspike interval adaptation, we calculated the ratio between the first and second interspike intervals. An adaptation ratio below 1.0 indicated that the second interspike interval was longer than the first interspike interval. Bursty neurons showed a significantly smaller ratio than non-bursty neurons (Fig. 3F; mean \pm SEM: bursty, $0.874 \pm 0.007$; non-bursty, 
$\left.0.986 \pm 0.005 ; W=32,401.5, p<10^{-15}\right)$. These results indicate that bursty and non-bursty neurons differ not only in their average interspike intervals but also in how their interspike intervals evolve during periods of high instantaneous firing rates.

Recent studies reported that putative grid cells showed some degree of anatomical clustering in the MEC (Burgalossi et al., 2011; Heys et al., 2014; Ray et al., 2014). We investigated whether we could find indirect evidence for an anatomical clustering of bursty and non-bursty neurons. We counted all pairs of cells recorded simultaneously on the same tetrode (Fig. 3G). Cell pairs could contain two bursty neurons, two non-bursty neurons, or one bursty and one non-bursty neuron. To determine the number of pairs that would be obtained by chance given the number of recorded neurons, we randomly reassigned class memberships of the neurons and counted the number of cell pairs obtained. This procedure was repeated 10,000 times to obtain a shuffled distribution to which the empirical data could be compared. The number of bursty cell pairs was higher than expected by chance (Fig. 3G; bursty, 277 pairs; mean shuffled distribution, 197.5; $\left.p<10^{-3}\right)$. The number of non-bursty cell pairs observed was not significantly different than what could be expected by chance (non-bursty, 160 pairs; mean shuffled distribution, 134.8; $p=0.06$ ). We observed significantly fewer mixed cell pairs than expected by chance (mixed, 222 pairs; mean shuffled distribution, 326.7; $\left.p<10^{-10}\right)$. These results suggest that bursty neurons show a higher degree of anatomical clustering than non-bursty neurons.

Our histological analysis showed that as we advanced the tetrodes between recording days, the recording sites became progressively more superficial. This allowed us to investigate whether the ratio of bursty to non-bursty neurons changed as more recording sites entered layer II. We compared the ratio of bursty to non-bursty neurons during the first and last three recording sessions of each mouse (Fig. $3 H$ ). There was no significant change in the ratio during the course of the experiment (Pearson's $\chi^{2}$ test, $\chi^{2}=2.8394, \mathrm{df}=5, p=0.72$ ). This suggests that bursty and non-bursty neurons are intermingled at different depths of the superficial layers. Similarly, we did not observe a change in the ratio of grid cells to head-direction cells (Pearson's $\chi^{2}$ test, $\chi^{2}=$ $0.1214, \mathrm{df}=1, p=0.73$ ).

\section{Bursty and non-bursty MEC neurons preferentially encode distinct spatial information}

We assessed whether bursty and non-bursty neurons have different spatial firing patterns. For this analysis, we considered the activity of MEC neurons during a 20 min trial of exploration in the open field. Examples of firing rate maps, spatial autocorrelations, and spike-time autocorrelations of grid cells are shown in Interspike interval.
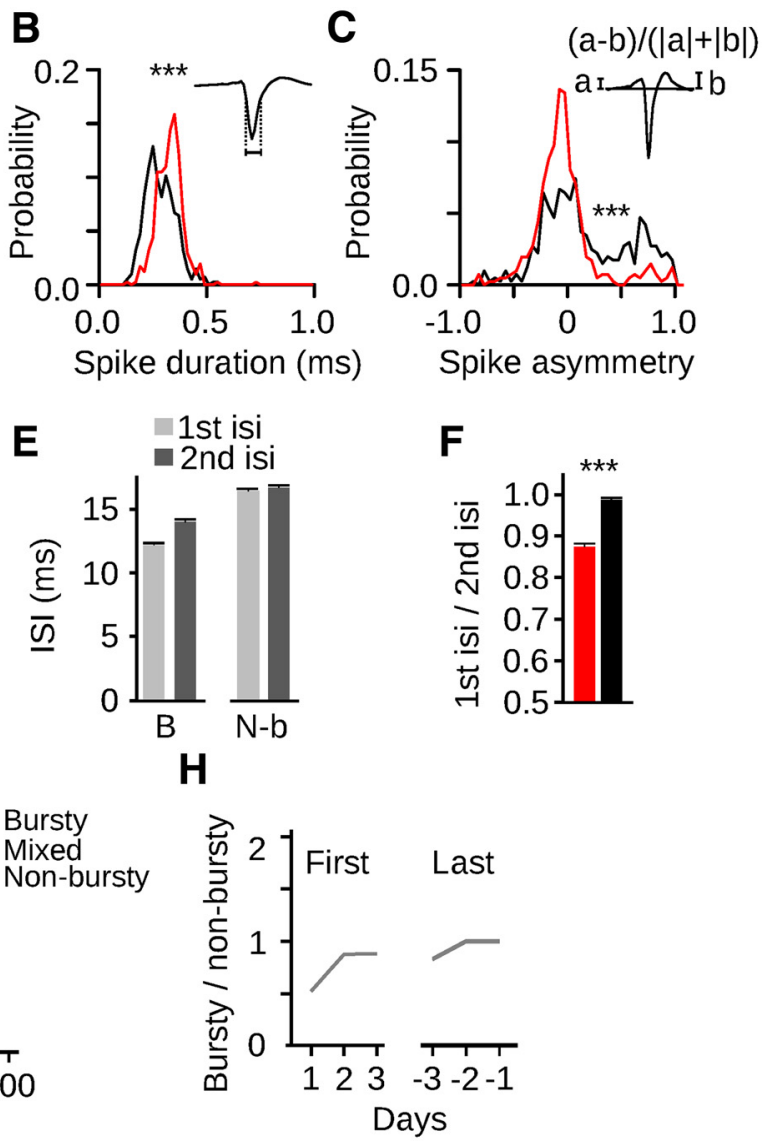

Figure 3. Spike waveform, interspike interval adaptation, and distribution across recording sites of bursty and non-bursty neurons. $\boldsymbol{F}$, Ratio of the first and second interspike intervals during spike triplets. $\boldsymbol{G}$, Clustering of bursty neurons around recording ursty neurons and fewer mixed pairs were observed than expected by chance. $\boldsymbol{H}$, Ratio of bursty to non-bursty neurons during the first 3 and the last 3 recording days. The ratio was relatively stable throughout the experiment. ${ }^{* *} p<10^{-3}{ }^{* * *} p<10^{-10}$. isi,

Figure $4 A$. Clear examples of grid cells were observed in both classes of neurons. However, examination of grid scores showed that they were significantly higher for bursty than for non-bursty neurons (Fig. 4B; median: bursty, 0.171; non-bursty, 0.037; Wilcoxon rank sum test, $\left.W=68,869, p<10^{-4}\right)$. Accordingly, the probability that a neuron was classified as a grid cell was higher for bursty than for non-bursty neurons [Fig. 4C; bursty, $25.85 \%$ (106 of 410); non-bursty, 13.79\% (56 of 406); Pearson's $\chi^{2}$ test, $\left.\chi^{2}=17.8981, \mathrm{df}=1, p<10^{-4}\right]$. This was also observed when we limited the analysis to tetrodes on which neurons of both groups were recorded simultaneously [bursty, 30.81\% (53 of 172); nonbursty, $18.38 \%$ ( 25 of 136); Pearson's $\chi^{2}$ test, $\chi^{2}=5.5666$, $\mathrm{df}=1$, $p=0.018]$. A spatial information score was calculated for each neuron. The spikes of bursty neurons contained more spatial information than those of non-bursty neurons (Fig. 4D; Wilcoxon rank sum test, $W=66,830, p<10^{-5}$ ), and the proportion of irregular spatially selective neurons was higher in bursty neurons than in non-bursty neurons [bursty, $29.56 \%$ (81 of 274); non-bursty, $13.74 \%$ (36 of 262); Pearson's $\chi^{2}$ test, $\chi^{2}=18.7317$, $\left.\mathrm{df}=1, p<10^{-4}\right]$. Importantly, when the analysis was limited to grid cells of both classes of neurons, bursty and non-bursty neurons still showed significantly different spike-time autocorrela- 
A
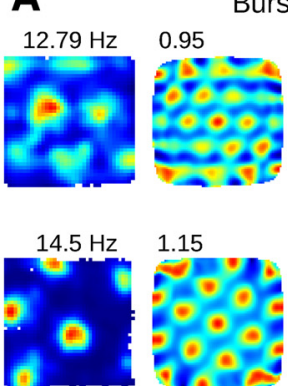

1.15
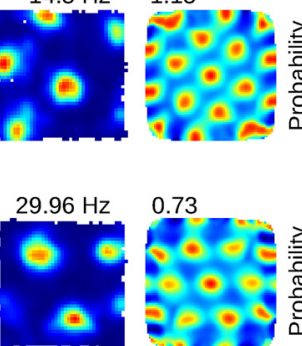

Max

B

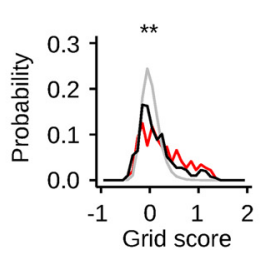

Bursty neurons
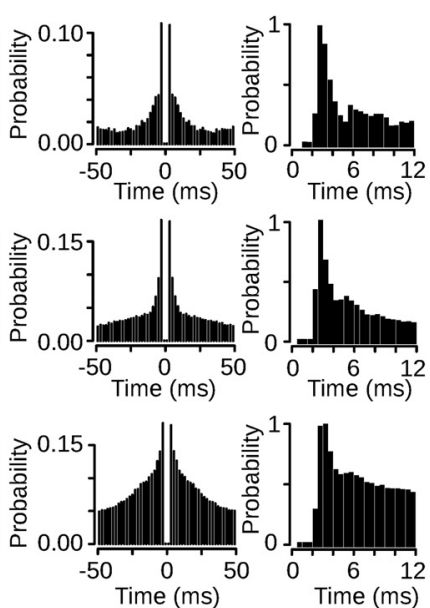

D

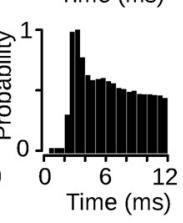

C

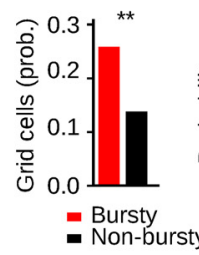

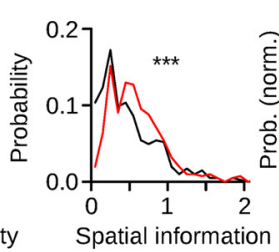

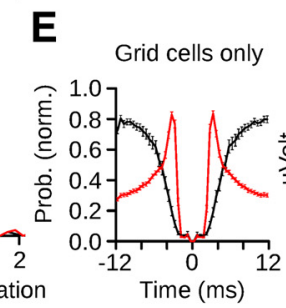

Non-bursty neurons
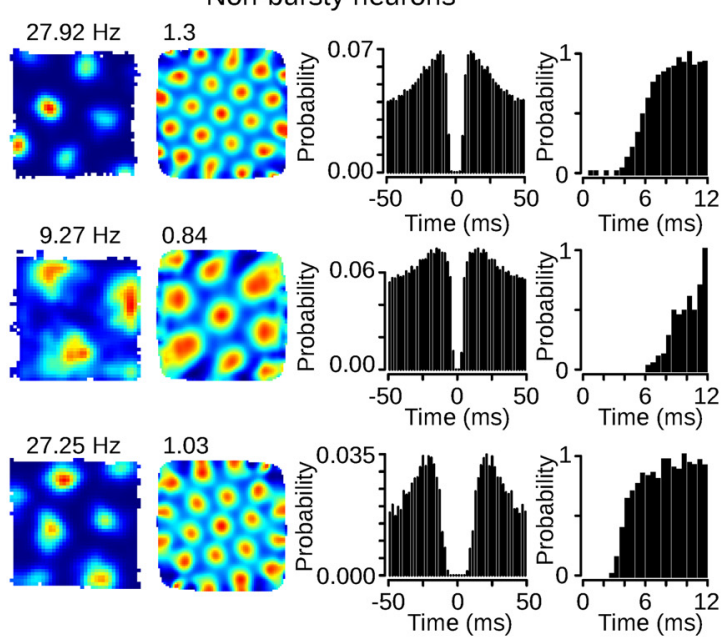

F

G

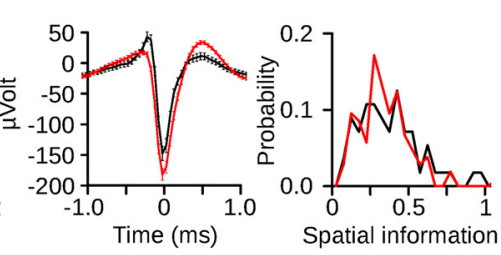

Figure 4. Grid cell spatial periodicity of bursty and non-bursty neurons. A, Firing rate maps, spatial autocorrelations, and spike-time autocorrelations at two different time scales for six neurons with grid periodicity. The three neurons on the left are bursty neurons, whereas those on the right are non-bursty neurons. $B$, Distribution of grid scores for bursty and non-bursty neurons. The gray line indicates the distribution expected by chance. C, Proportion of grid cells for bursty and non-bursty neurons. D, Distribution of spatial information scores for both classes of neurons. $\boldsymbol{E}-\boldsymbol{G}$, Mean spike-time autocorrelation, mean spike waveform, and distribution of spatial information scores for bursty and non-bursty grid cells. ${ }^{* *} p<10^{-4} ;{ }^{* * *} p<10^{-5}$.

tions and spike waveforms (Fig. 4E,F). Grid cells of the two classes show no significant difference in spatial information (Fig. $4 G$; Wilcoxon rank sum test, $W=3272, p=0.285$ ).

Next, we tested whether the head direction of the animal was differently encoded by bursty and non-bursty neurons. Figure $5 \mathrm{~A}$ shows examples of neurons with head-direction tuning for both groups. Comparison of head-direction vector length indicated stronger head-direction tuning in non-bursty neurons (Fig. 5B; median: bursty, 0.13; non-bursty, 0.21 ; Wilcoxon rank sum test, $W=46,036, p<10^{-7}$ ). Of 536 recorded neurons, 88 neurons were classified as head-directional cells (mean vector length $>0.4$ ). The probability that non-bursty neurons were classified as head-direction cells was higher than that of bursty neurons [Fig. 5C; bursty, $5.11 \%$ (14 of 274); non-bursty, $28.24 \%$ (74 of 262); Pearson's $\chi^{2}$ test, $\chi^{2}=50.5657, \mathrm{df}=1, p<10^{-11}$. This was also true when we considered only tetrodes on which both types of neurons were recorded at the same time [bursty, $6.61 \%$ ( 8 of 121 ); non-bursty, $22.77 \%$ (23 of 101); Pearson's $\chi^{2}$ test, $\chi^{2}=$ 10.6597, $\mathrm{df}=1, p=0.001]$.

The difference in head-direction tuning was not simply caused by the difference in spatial information score between the neurons of the two cell classes (Fig. 5D). The difference was present when considering only neurons with a low or high spatial information score (spatial information $<0.48$; mean \pm SEM: bursty, $0.134 \pm 0.011$; non-bursty, $0.266 \pm 0.019$; Wilcoxon rank sum test, $W=10,884, p<10^{-4}$; spatial information $>0.48$; mean \pm SEM: bursty, $0.188 \pm 0.011$; non-bursty, $0.333 \pm 0.024$; $\left.W=12,266, p<10^{-5}\right)$. Interestingly, head-direction selectivity was negatively correlated with grid score (Pearson correlation, $r=-0.26 ; p<10^{-9}$ ), and there was no significant difference in head-direction selectivity between the two classes when only grid cells were considered (Fig. 5E; mean \pm SEM, grid cells: bursty, $0.128 \pm 0.009$; non-bursty, $0.163 \pm 0.026$; Wilcoxon rank sum test, $W=1607, p=0.85$; non-grid cells: bursty, $0.180 \pm 0.010$; non-bursty, $0.323 \pm 0.017$; Wilcoxon rank sum test, $W=29,020$, $\left.p<10^{-8}\right)$. Only four conjunctive grid $\times$ head-direction cells were detected, and all were non-bursty neurons. More conjunctive cells were detected when the threshold for grid periodicity and head directionality were lowered to 0.3 , and 21 of 29 conjunctive cells were non-bursty neurons.

The MEC also contains a small population of border cells that fire only when the animal is near a border of the recording environment (Savelli et al., 2008; Solstad et al., 2008; Bjerknes et al., 2014). Examples of bursty and non-bursty neurons that fired along a border are shown in Figure 6A. Non-bursty neurons were more likely to encode border proximity than bursty neurons. Border scores were higher for non-bursty than for bursty neurons (Fig. 6B; median: bursty, 0.010; non-bursty, 0.278; Wilcoxon rank sum test, $\left.W=103,693.5, p<10^{-9}\right)$. Moreover, border cells were more frequently observed in non-bursty than in bursty neurons even when we removed grid cells from the analysis [Fig. 6C; bursty, $12.58 \%$ (38 of 302); non-bursty, $20.11 \%$ (70 of 348); Pearson's $\chi^{2}$ test, $\chi^{2}=6.0884, \mathrm{df}=1, p=0.014$ ] or limited the analysis to tetrodes on which neurons from both groups were recorded simultaneously [bursty, 8.55\% (13 of 152); non-bursty, $19.08 \%$ (25 of 131); Pearson's $\chi^{2}$ test, $\chi^{2}=5.8376, \mathrm{df}=1, p=$ $0.016]$. To investigate the potential relationship between headdirection selectivity and border score, we plotted these two variables against each other (Fig. $6 D$ ). We found no evidence that the difference in border scores between bursty and non-bursty neurons was caused by a difference in head-direction selectivity. Indeed, cells with a high border score could have high or low 
A

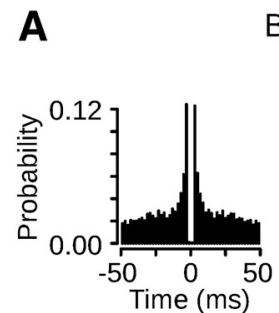

\section{Bursty neurons}
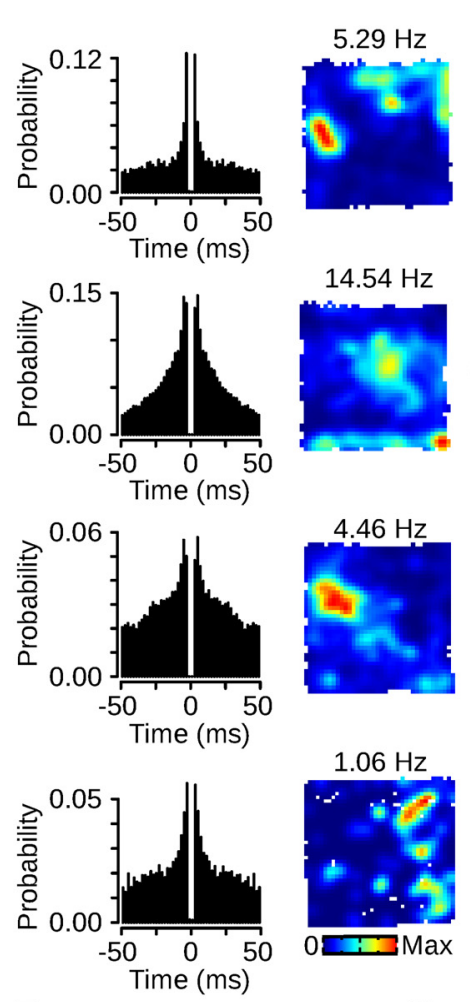

B
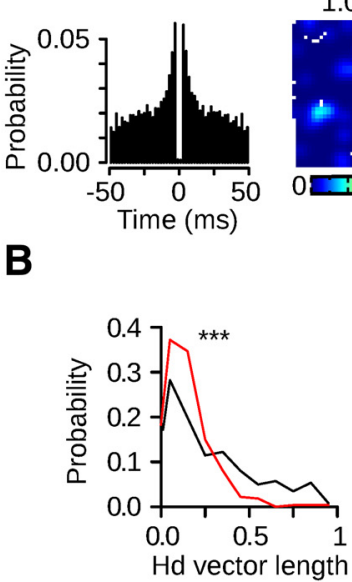

C

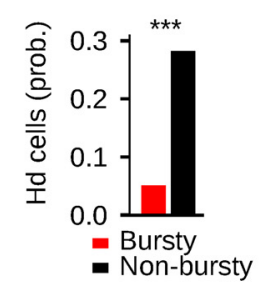

$4.11 \mathrm{~Hz}$

270

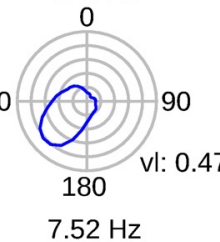

0

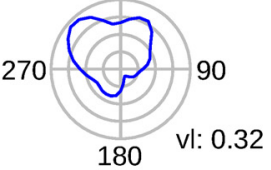

$1.62 \mathrm{~Hz}$

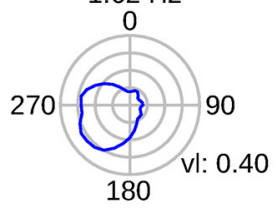

$0.48 \mathrm{~Hz}$
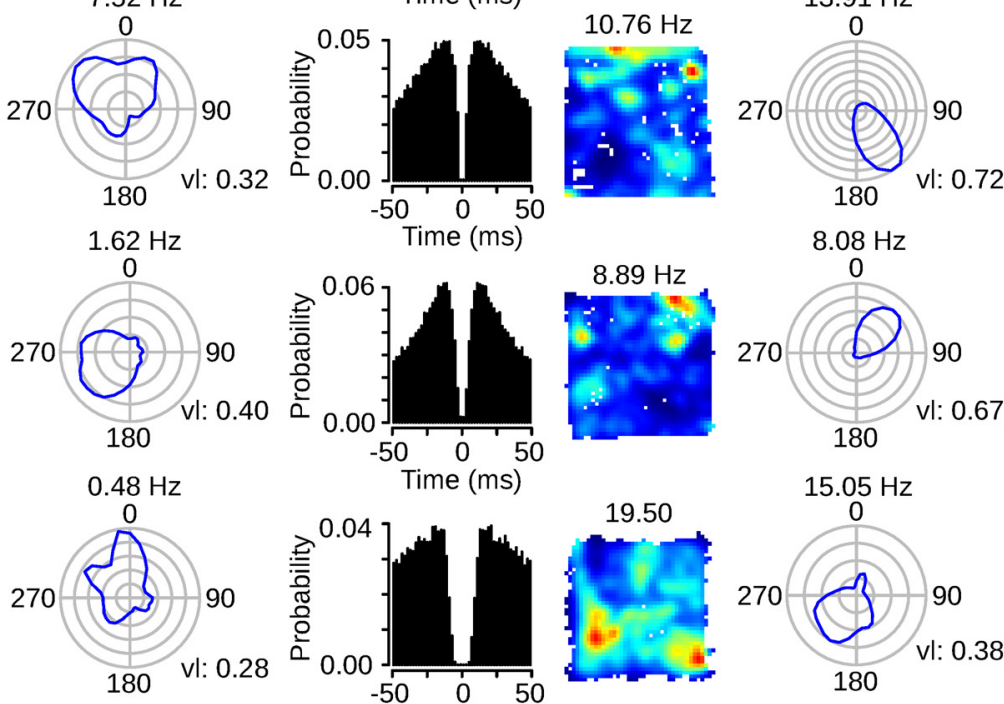

$8.08 \mathrm{~Hz}$

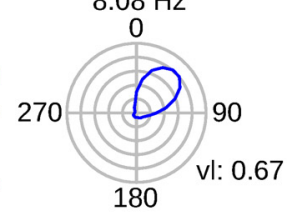

19.50
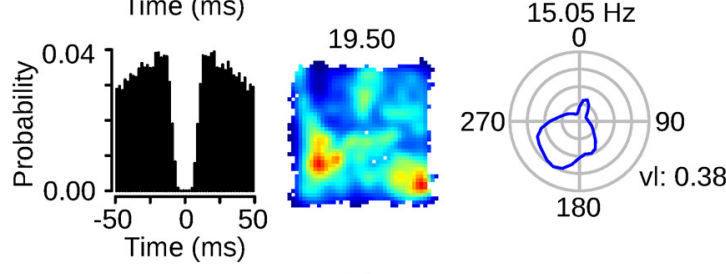

E
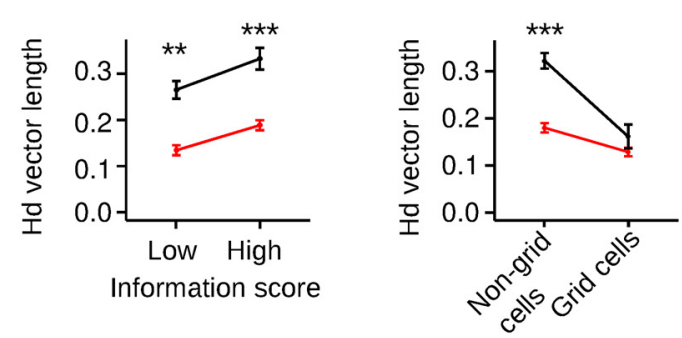

Figure 5. Head-direction selectivity is stronger in non-bursty than in bursty neurons. $\boldsymbol{A}$, Spike-time autocorrelations, firing rate maps, and head-direction rate polar plots of four bursty and four non-bursty neurons. The peak firing rate is indicated above each firing rate map and head-direction polar plot. vl, Mean vector length of the head-direction polar plot. $\boldsymbol{B}$, Distribution of head-direction mean vector length for bursty and non-bursty neurons. $C$, Proportion of head-direction cells in bursty and non-bursty neurons. $\boldsymbol{D}$, Head-direction vector length for bursty and non-bursty neurons with high $(>0.48)$ and low $(<0.48)$ spatial information scores. $\boldsymbol{E}$, Head-direction vector length for neurons with and without grid cell spatial periodicity. Grid cells had lower head-direction selectivity. ${ }^{* *} p<10^{-4}$

head-direction selectivity, and the correlation between these two variables was weak (Pearson correlation, $r=0.23, p<10^{-6}$ ). Together, these results uncover a dissociation between bursty and non-bursty neurons in regard to the spatial signals that they encode. Grid cell periodicity is more common in bursty neurons, whereas head-direction selectivity and border encoding are more frequent in non-bursty neurons (Fig. 6E).

\section{Different theta firing properties of bursty and non-bursty MEC neurons}

Theta oscillations $(6-10 \mathrm{~Hz})$ contribute to the encoding of spatial information and provide a common reference frame for communication between the entorhinal cortex and the hippocampus (Hafting et al., 2008; Huxter et al., 2003, 2008; Mizuseki et al., 2009; Brandon et al., 2011). We therefore investigated whether the activity of bursty and non-bursty neurons was differentially modulated by theta oscillations associated with active exploration. We first compared the preferred theta phase of neurons in the two classes. Most bursty and non-bursty neurons fired before the trough of locally recorded theta oscillations (Fig. 7A). However, bursty neurons tended to discharge before non-bursty neurons (Fig. $7 B$; mean \pm SEM: bursty, $33.88 \pm 2.00^{\circ}$; non-bursty, $49.23 \pm 4.05^{\circ}$; permutation test, $p<10^{-3}$ ). This difference in preferred firing phase was still present when the analysis was limited to tetrodes on which neurons from both groups were recorded simultaneously (mean \pm SEM: bursty, $35.13 \pm 3.04^{\circ}$; non-bursty, $46.2 \pm 7.99^{\circ}$; permutation test, $p=0.05$ ).

The strength of theta modulation for a neuron was estimated by calculating the mean vector length of the theta phase of the spikes. We found that the shape of the mean vector length distributions was significantly different between the bursty and nonbursty neurons (Fig. 7C; two-sample Kolmogorov-Smirnov test, $\left.D=0.1794, p<10^{-5}\right)$. The medians of the two distributions, however, were not significantly different (Wilcoxon rank sum test, $W=86,154, p=0.385$ ). The distribution of the mean vector of non-bursty neurons was bimodal (Fig. 7C; Silverman's test 

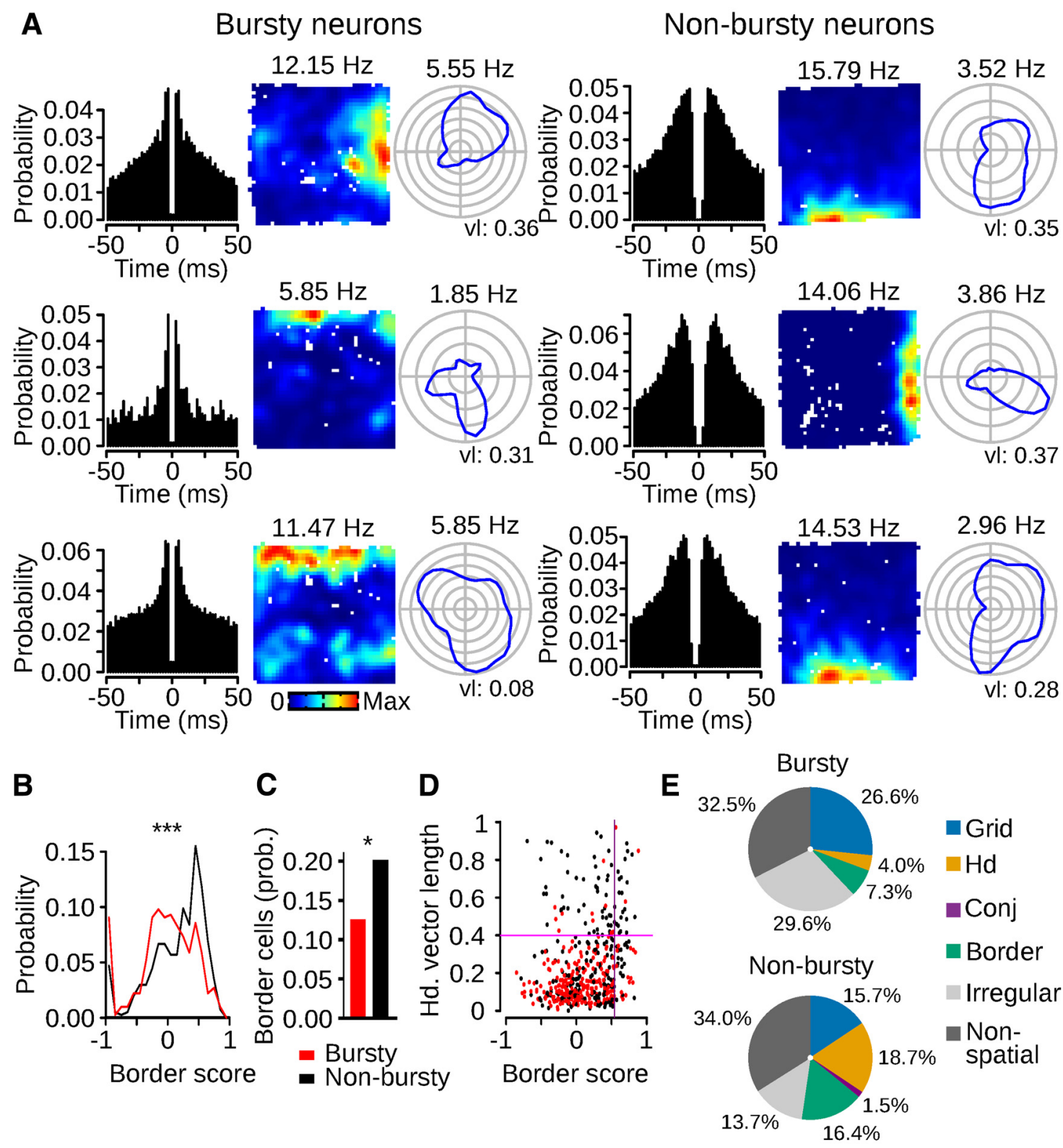

Figure 6. Border proximity preferentially encoded by non-bursty neurons. $A$, Spike-time autocorrelations, firing rate maps, and head-direction polar plots of six neurons firing predominantly along one of the environment walls. vl, Mean vector length of the head-direction polar plot. $\boldsymbol{B}$, Distribution of border scores for bursty and non-bursty neurons. $\boldsymbol{C}$, Proportion of border cells in bursty and non-bursty neurons. $\boldsymbol{D}$, Head-direction vector length plotted against the border score. $\boldsymbol{E}$, Percentages of the different spatially selective cell types in bursty and non-bursty neurons. Grid, Grid cells (grid score $>0.54$ ); $H d$, head-direction cells (Hd vector length $>0.4$ ); Conj, conjunctive grid $\times$ head-direction cells (grid score $>0.54$; Hd vector length $>0.4$ ); Border, border cells (border score $>0.528$; grid score $<0.54$ ); Irregular, irregular spatially selective cells (spatial information $>0.48$; grid score $<0.54$; Hd vector length $<0.4$ ); Non-spatial, nonspatially selective cell (spatial information $<0.48$; grid score $<0.54$; Hd vector length $<0.4$ ). The data of four mice for which head direction was not available were not included. ${ }^{*} p<0.05$; ${ }^{* * *} p<10^{-9}$.

with $k=1, p=0.01)$. When comparing subgroups of non-bursty neurons with weak and strong theta modulation (cutoff $=0.35$ ), we found a small but significant difference in the mean firing rate (median: weak, $1.89 \mathrm{~Hz}$; strong, $1.53 \mathrm{~Hz} ; W=23,975, p=$ $0.0026)$. However, the two subgroups had similar spike waveforms and spatial information scores. The proportion of grid cells appeared higher in weakly theta-modulated non-bursty neurons [weak, 17.57\% (39 of 222); strong, 9.24\% (17 of 184); Pearson's $\chi^{2}$ test, $\chi^{2}=5.1895, p=0.023$ ]. In contrast, head-direction cells were more frequently observed in strongly theta-modulated non-bursty neurons [weak, 19.31\% (28 of 145); strong, 39.32\% (46 of 117); Pearson's $\chi^{2}$ test, $\chi^{2}=11.8193, p<10^{-3}$ ]. These differences were not present for bursty neurons when we used the same threshold for theta modulation [grid cells, bursty: weak,
$28.47 \%$ (78 of 274); strong, 20.59\% (28 of 136); Pearson's $\chi^{2}$ test, $\chi^{2}=2.5466, p=0.11$; head-direction cells: weak, $2.92 \%$ (8 of 185); strong, 4.4\% (6 of 89); Pearson's $\chi^{2}, \chi^{2}=0.7242, p=0.55$ ].

MEC neurons show intrinsic theta frequencies that differ from the frequency of theta oscillations recorded in the local field potentials (Hafting et al., 2008; Schmidt-Hieber and Häusser, 2013). The intrinsic theta frequency of each neuron was estimated from the power spectrum of the instantaneous firing rate (Fig. 7D). Bursty neurons had a significantly higher intrinsic theta frequency (Fig. 7E; mean \pm SEM: bursty, $8.32 \pm 0.025 \mathrm{~Hz}$; non-bursty, $8.12 \pm 0.027$; Wilcoxon rank sum test, $W=$ $\left.42,464.5, p<10^{-7}\right)$. The theta rhythmicity index calculated from these power spectra also indicated that the spiking activity of bursty neurons was more rhythmic at theta frequency than that of 
non-bursty neurons (Fig. 7F; median: bursty, 0.122 ; non-bursty, $0.089 ; W=$ $63,589, p<10^{-8}$ ).

The firing rate modulation of neurons by theta oscillations was also different in the two groups of neurons. The firing probability as a function of theta phase is shown in Figure 7G. One noticeable difference between the groups is the presence of a secondary peak between phase $180^{\circ}$ and $270^{\circ}$ for bursty neurons. The proportion of neurons with a bimodal theta firing rate histogram was indeed higher for bursty than non-bursty neurons [bursty, $23.66 \%$ (97 of 410 ); non-bursty, $5.17 \%$ (21 of 406); Pearson's $\chi^{2}$ test, $\chi^{2}=$ $\left.54.8733, \mathrm{df}=1, p=<10^{-12}\right]$.

We divided the neurons of the two groups into spatially defined cell classes and compared their theta modulation (Fig. 7H). Surprisingly, theta vector lengths of head-direction cells were larger than those of grid cells (median: grid cells, 0.216 ; head-direction cells, 0.438 ; Wilcoxon rank sum test, $W=4821, p<$ $\left.10^{-3}\right)$. This difference was also detected when only non-bursty cells were considered (Fig. $7 \mathrm{H}$; non-bursty median: grid cells, 0.207; head-direction cells, 0.444 ; $W=1225, p=0.002$ ), but not within bursty neurons. The absence of difference for bursty neurons might be related to the low number of head-direction cells in this class $(n=14)$. There was no difference in theta modulation between other spatially defined cell types or the two neuronal classes (bursty vs non-bursty). Together, the differences in preferred theta phase, theta vector length, theta rhythmicity, and intrinsic theta frequency all suggest that bursty and non-bursty neurons could perform distinct functions during theta oscillations.

The firing rate of stellate and pyramidal cells in layer II of the MEC is also differentially modulated by theta oscillations (Ray et al., 2014), and this difference can be used to identify putative stellate and pyramidal cells from unidentified neurons recorded extracellularly (Tang et al., 2014). We used a similar classifier to investigate how the bursty/ non-bursty classification relates to stellate and pyramidal cells. The classifier was applied only to neurons recorded during the last five recording sessions of each mouse to restrict the analysis to a subset of neurons in which most were located in layer II. This subset represented $37.0 \%$ of the entire data set ( 302 of 816 neurons). Figure 7 I shows how neurons recorded in the current study were grouped into putative calbindin $^{+}\left(\mathrm{pCalb}^{+}\right.$, pyramidal cells) and putative calbindin ${ }^{-}$ ( $\mathrm{pCalb}^{-}$, stellate cells) cells by the classifier. Of 302 neurons, 13 were in the guarding zone and were not assigned a cell type. According to the classifier, 172 (59.52\%) neurons were pCalb ${ }^{-}$and 117 (40.48\%)

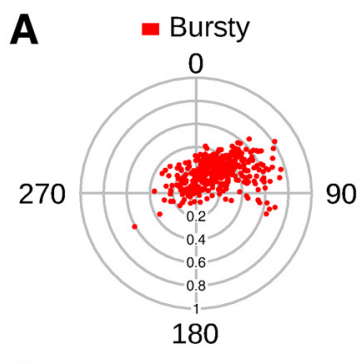

C

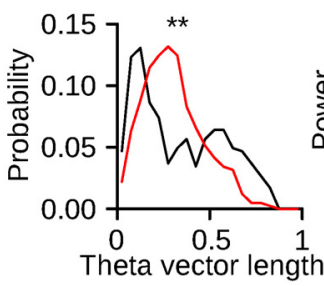

G

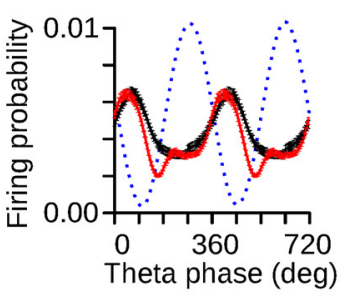

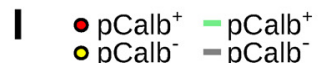

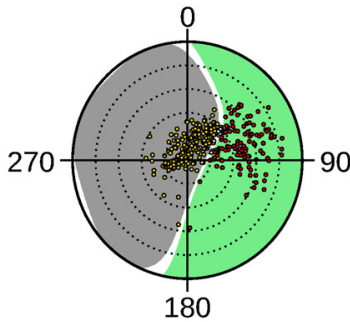

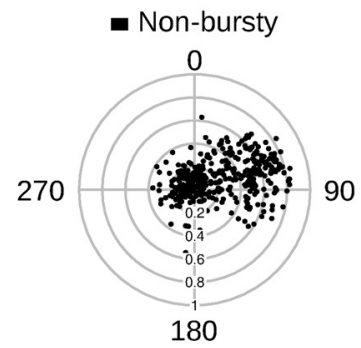

E
B

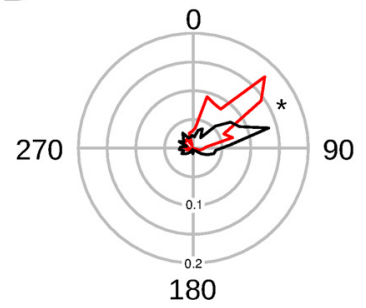

F
Figure 7. Different temporal properties of bursty and non-bursty neurons during theta oscillations. $A$, Polar plots showing the preferred theta phase and theta mean vector length of bursty and non-bursty neurons. $B$, Probability distribution of preferred theta phase for bursty and non-bursty neurons. $\boldsymbol{C}$, Distribution of theta vector length for bursty and non-bursty neurons. Asterisks indicate that the two distributions were significantly different. There was no significant difference between the medians of the two groups (Wilcoxon rank sum test, $W=86,154, p=0.385$ ). D, Power spectra of the instantaneous firing rate of bursty and non-bursty neurons. $\boldsymbol{E}$, Mean theta peak frequency of bursty and non-bursty neurons. $\boldsymbol{F}$, Distribution of the theta rhythmicity index. $\boldsymbol{G}$, Firing probability as a function of theta phase for both classes of neurons. $\boldsymbol{H}$, Theta vector length of bursty and non-bursty neurons divided into different spatially defined cell types. Grid, Grid cells (grid score $>0.54$ ); Hd, head-direction cells (Hd vector length $>0.4$ ); Conj, conjunctive grid $\times$ head-direction cells (grid score $>0.54$; Hd vector length $>0.4$ ); Border, border cells (border score $>0.528$; grid score $<0.54$ ); Irregular, irregular spatially selective cells (spatial information $>0.48$; grid score $<0.54$; Hd vector length $<0.4$ ); Non-spatial, nonspatially selective cell (spatial information $<0.48$; grid score $<0.54$; $\mathrm{Hd}$ vector length $<0.4$ ); Int, interneurons (mean firing rate $>10 \mathrm{~Hz}$ ). I, Classification of neurons as putative calbindin ${ }^{+}\left(\mathrm{pCalb}^{+}\right)$and calbindin $^{-}$(pCalb $^{-}$) cells based on their preferred theta phase and coupling strength. The data from Tang et al. (2014) were used to construct the classifier. J, Left, Proportion of neurons falling into the four possible cell categories (pCalb ${ }^{+} / \mathrm{bursty}, \mathrm{pCalb} \mathrm{b}^{+} / \mathrm{non}^{-}$ bursty, $\mathrm{pCalb}^{-}$/bursty, $\mathrm{pCalb}{ }^{-}$/non-bursty). Right, Proportion of $\mathrm{pCalb}^{-}$and $\mathrm{pCalb}^{+}$neurons that were grid cells, headdirection cells, and border cells. ${ }^{*} p<0.01 ;^{* *} p<10^{-5} ;{ }^{* * *} p<10^{-8}$. 

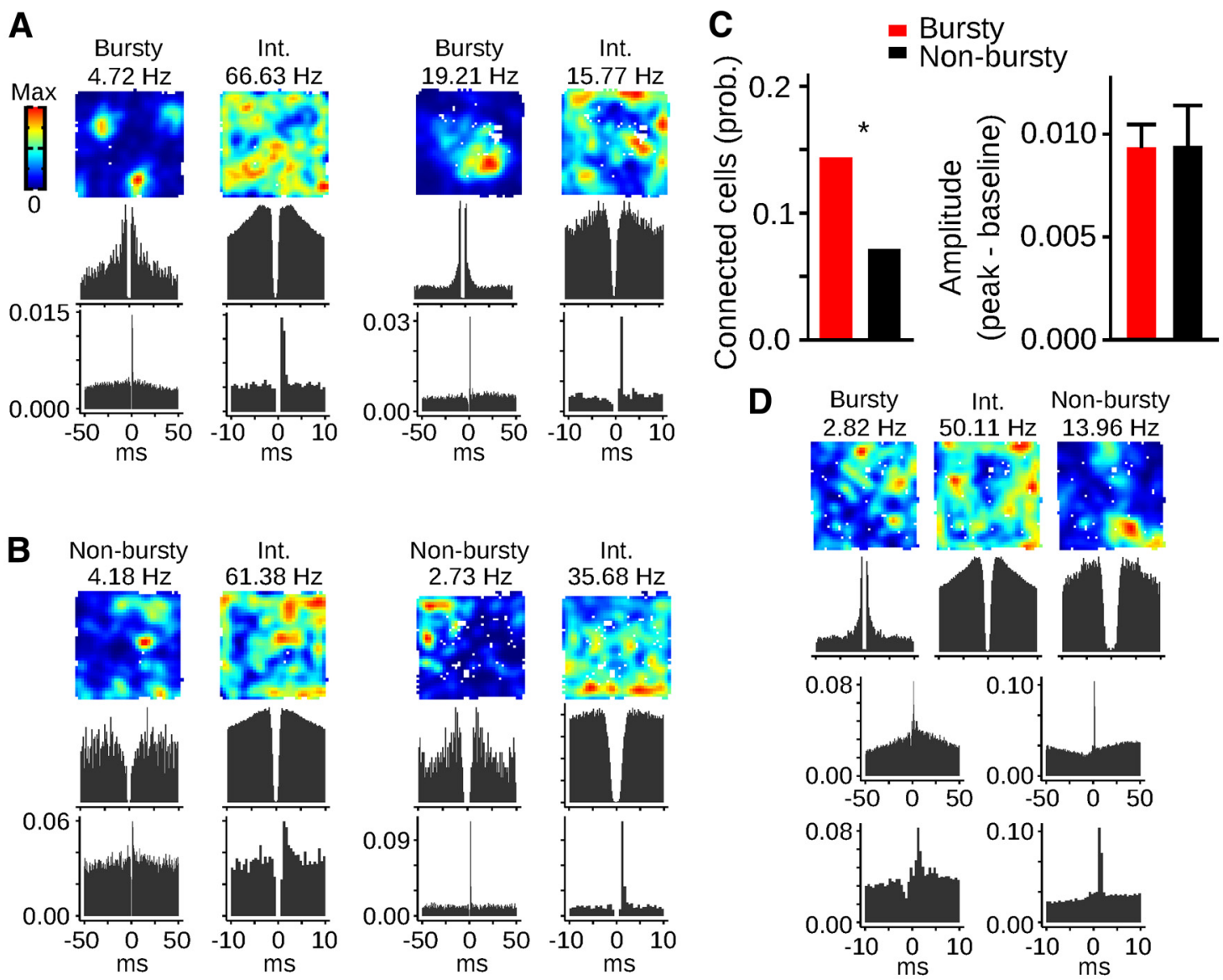

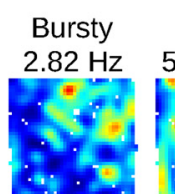

Int. Non-bursty
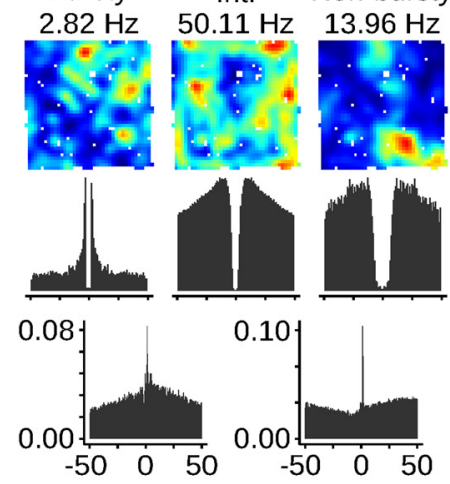

0.10
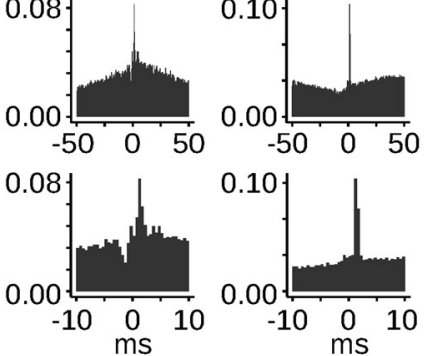

Figure 8. Spike transmission from excitatory neurons to putative interneurons preferentially involves bursty neurons. $A$, Two examples of putative connection between a bursty neuron and an interneuron. Top two rows, Firing rate maps and spike-time autocorrelations of the bursty neurons and interneurons. Numbers above the maps indicate peak firing rates. Bottom row, Spike-time crosscorrelations at two different time scales between the bursty neurons and interneurons. The sharp peaks ( $>5$ SDs) with a latency between 1 and 4 ms suggest the presence of excitatory connectivity between the neurons. The lower bins near time 0 in the crosscorrelations are attributable to a refractory period in the spike detection algorithm and affect only pairs of neurons recorded on the same tetrode. $\boldsymbol{B}$, Same as in $\boldsymbol{A}$ but for two pairs of non-bursty neurons and interneurons. $\boldsymbol{C}$, Left, Probability that neurons excite (significant peak in crosscorrelation) other neurons, shown separately for bursty and non-bursty neurons. Right, Mean amplitude of peaks (peak - baseline) at a short latency, shown for bursty and non-bursty neurons. Mean \pm SEM. $\boldsymbol{D}$, Crosscorrelation of a bursty (left) and a non-bursty (right) neuron with the same putative interneuron (middle). ${ }^{*} p<0.01$. Int., Interneurons.

$\mathrm{pCalb}^{-}$rather than as $\mathrm{pCalb}^{+}$, but the difference did not reach significance [Fig. 7J; pCalb $^{-}$and grid, 25.58\% (44 of 172); $\mathrm{pCalb}^{+}$and grid, 16.24\% (19 of 117); Pearson's $\chi^{2}$ test, $\chi^{2}=$ $3.038, p=0.08$ ]. However, grid scores were larger for $\mathrm{pCalb}^{-}$ than for $\mathrm{pCalb}^{+}$neurons (median: $\mathrm{pCalb}^{-}, 0.184$; $\mathrm{pCalb}^{+}$, $0.044 ; W=11,945, p<0.01)$. The percentage of head-direction cells within $\mathrm{pCalb}^{+}$and $\mathrm{pCalb}^{-}$neurons was not significantly different [Fig. 7J; pCalb ${ }^{-}$and Hd, 13.11\% (16 of 122); pCalb $^{+}$ and Hd, $19.28 \%$ (16 of 83); Pearson's $\chi^{2}$ test, $\chi^{2}=0.9946, \mathrm{df}=1$, $p=0.32$. Similarly, border cells were equally distributed among pCalb $^{+}$and pCalb ${ }^{-}$cells [Fig. 7J; pCalb ${ }^{-}$and border, $13.95 \%$ (24 of 172); $\mathrm{pCalb}^{+}$and border, $17.09 \%$ (20 of 117); Pearson's $\chi^{2}$ test, $\left.\chi^{2}=0.3166, \mathrm{df}=1, p=0.57\right]$. Using the same subset of neurons, grid cells, head-direction cells, and border cells were still differentially distributed between bursty and non-bursty neurons [grid cells: bursty, 31.21\% (44 of 141); non-bursty, 12.84\% (19 of 148 ); Pearson's $\chi^{2}$ test, $\chi^{2}=13.2333$, $\mathrm{df}=1, p<10^{-3}$; headdirection cells: bursty, 4.76\% (5 of 105); non-bursty, 27.0\% (27 of 100); Pearson's $\chi^{2}$ test, $\chi^{2}=17.5773, \mathrm{df}=1, p<10^{-4}$; border cells: bursty, $8.51 \%$ (12 of 141); non-bursty, $21.62 \%$ (32 of 148); Pearson's $\chi^{2}$ test, $\chi^{2}=8.6278, \mathrm{df}=1, p<0.01$ ]. Thus, for our data set, the bursty/non-bursty classification appeared to be a better predictor for the type of spatial selectivity expressed by a neuron than its $\mathrm{pCalb}^{+} / \mathrm{pCalb}^{-}$assignation (Fig. $7 J$ ).
Bursty and non-bursty neurons' functional connectivity with fast-spiking interneurons

If bursty and non-bursty neurons constitute functionally distinct classes of neurons, their connectivity to other neurons in the network might also differ. To test this, we calculated spike-time crosscorrelograms of simultaneously recorded neurons. A high peak $(>5$ SDs above baseline) within a short latency $(<4 \mathrm{~ms})$ in the crosscorrelogram was treated as indicative of a putative excitatory connection between the cells (Csicsvari et al., 1998; Marshall et al., 2002; Maurer et al., 2006; Mizuseki et al., 2009; Dupret et al., 2013). Examples of four spike-time crosscorrelations with a high peak at short latency are shown in Figure 8, $A$ and B. Of 4873 tested crosscorrelations, $94(1.93 \%)$ showed a peak at short latency.

Analysis of the pairs of neurons with a significant peak at short latency indicated that the putative postsynaptic cells had a higher mean firing rate (mean \pm SEM: presynaptic neurons, $2.23 \pm 0.16$ $\mathrm{Hz}$; postsynaptic neurons, $21.17 \pm 1.94 \mathrm{~Hz}$; Wilcoxon rank sum test, $W=5651.5, p<10^{-15}$ ) and a narrower spike waveform (mean \pm SEM: presynaptic neurons, $0.322 \pm 0.005 \mathrm{~ms}$; postsynaptic neurons, $0.248 \pm 0.007 \mathrm{~ms}$; Wilcoxon rank sum test, $W=$ 1092.5, $p<10^{-12}$ ). These two findings are consistent with the notion that an important proportion of the putative postsynaptic neurons are parvalbumin-expressing interneurons (Royer et al., 2012; Buetfering et al., 2014). However, we did observe few pu- 
tative connections (12 of 94) in which the postsynaptic neuron had been classified as bursty or non-bursty (bursty onto bursty, 5 of 12; bursty onto non-bursty, 3 of 12; non-bursty onto bursty, 2 of 12; non-bursty onto non-bursty, 2 of 12; Pearson's $\chi^{2}$ test, $\chi^{2}$ $=0, \mathrm{df}=1, p=1$ ).

We calculated the probability that a bursty or non-bursty neuron was a putative presynaptic neuron of an excitatory connection. We found that in $68.09 \%$ of the spike-time crosscorrelations with a peak at short latency, the reference spikes were from a bursty neuron [Fig. 8C; bursty, 14.39\% (59 of 410); non-bursty, $7.14 \%$ (29 of 406); Pearson's $\chi^{2}$ test, $\chi^{2}=10.2273$, df $=1, p=$ $0.0014]$, indicating that bursty neurons were more likely to be presynaptic neurons than non-bursty neurons. The peak amplitude (peak - baseline) of the crosscorrelations was not different between connected pairs with a bursty or non-bursty neuron as a presynaptic cell (Fig. 8C; mean \pm SEM: bursty, $0.0094 \pm 0.001$; non-bursty, $0.0094 \pm 0.002$; Wilcoxon rank sum test, $W=963$, $p=0.83)$.

It has previously been shown that burst firing increases spike transmission efficacy (Csicsvari et al., 1998). Therefore, the larger number of putative monosynaptic connections involving bursty neurons could be attributable to their burst firing. To test this, we recalculated the spike-time crosscorrelations considering only presynaptic spikes that had not been preceded by another spike from the same neuron during the previous $15 \mathrm{~ms}$. Furthermore, we decreased the time window for the latency from $4 \mathrm{~ms}$ to $3 \mathrm{~ms}$. With these new parameters, we still found twice as many presynaptic bursty neurons compared with non-bursty neurons [bursty, 8.0\% (33 of 410); non-bursty, 3.0\% (12 of 406); Pearson's $\chi^{2}$ test, $\left.\chi^{2}=9.8, \mathrm{df}=1, p=0.0017\right]$.

We next analyzed whether postsynaptic neurons that were coupled to either bursty or non-bursty neurons showed different firing properties. To prevent differences between bursty and nonbursty neurons from influencing this analysis, we removed any pair in which the putative postsynaptic cell belonged to one of the two classes. We found that postsynaptic neurons differed in the modulation of their firing by theta oscillations. Indeed, postsynaptic neurons targeted by bursty neurons $(n=37)$ exhibited a longer theta vector length than neurons targeted by non-bursty cells ( $n=21$; median: target of bursty neurons, 0.48 ; target of non-bursty neurons, 0.23 ; Wilcoxon rank sum test, $W=189, p<$ $\left.10^{-3}\right)$. However, we did not detect a difference in mean firing rate (Wilcoxon rank sum test, $W=383 p=0.94$ ). Interestingly, we found evidence that the same putative postsynaptic neuron can be targeted by both bursty and non-bursty neurons (Fig. 8D).

\section{Bursty and non-bursty neuron dichotomy in a different species}

Finally, we asked whether the two populations of neurons described in this study are also present in other species. For this purpose, we analyzed the activity of MEC neurons recorded in the superficial layers of the MEC in behaving rats (Mizuseki et al., 2009, 2013). An advantage of this database was that each neuron was assigned to a cortical layer based on the locations at which the spike waveform was recorded on the silicon probes. The spiketime autocorrelation of neurons from layers II and III showed a similar clustering to that observed in mice (Fig. 9A-C). Bursty and non-bursty neurons were observed both in layers II and III, although the proportion of bursty neurons was higher in layer II than in layer III [Fig. 9B; layer II: bursty, 39.3\% (141 of 359); non-bursty, $60.7 \%$ (218 of 359); layer III: bursty, $18.6 \%$ (24 of 129); non-bursty, $81.4 \%$ (105 of 129); Pearson's $\chi^{2}$ test, $\chi^{2}=$ 17.2076, $\mathrm{df}=1, p<10^{-4}$. The mean spike-time autocorrela-

\section{A Layer II Layer III B \\ - Bursty $\quad$ Bursty \\ - Non-Bursty $\Delta$ Non-Bursty}
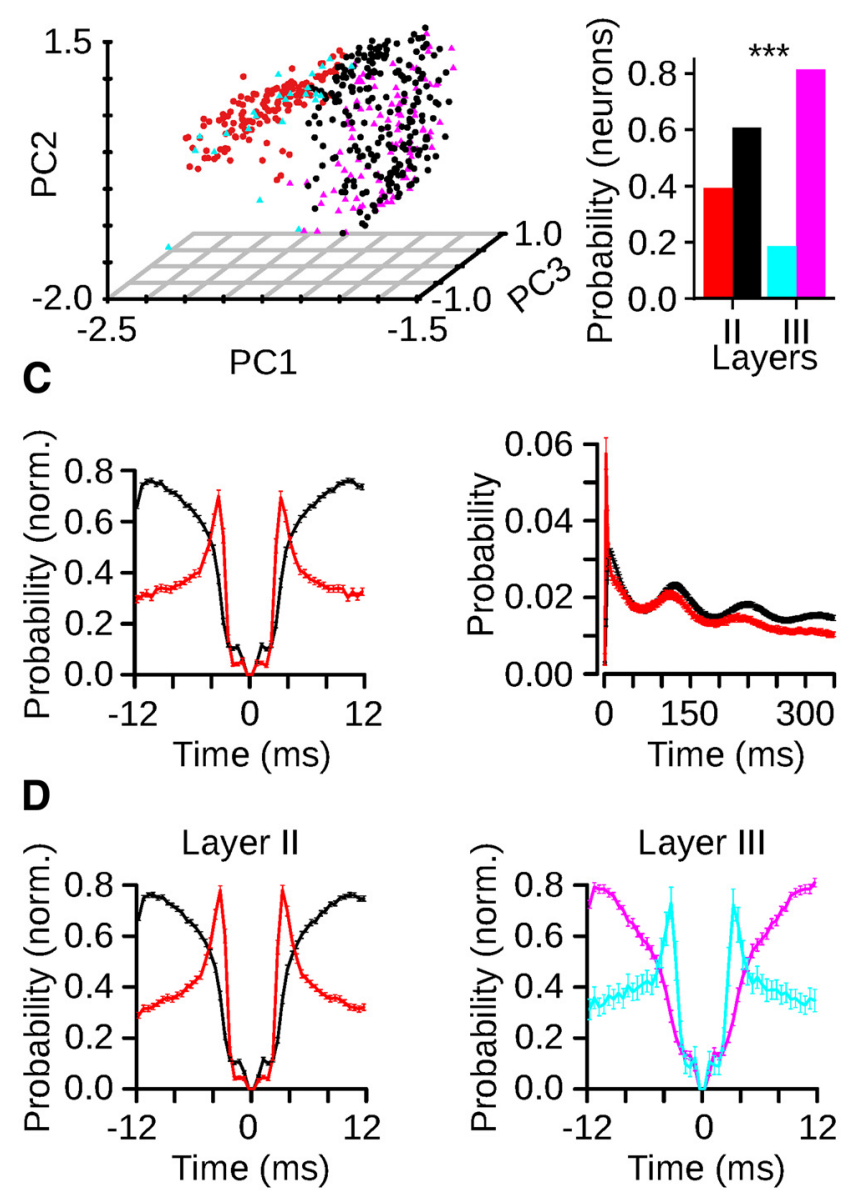

Figure 9. Distribution of bursty and non-bursty neurons in layers II and III of rats. A, A 3D scatter plot of the first three principal components of the spike-time autocorrelations (first 12 $\mathrm{ms})$. $k$-means clustering $(k=2)$ was used to assign the class membership of neurons. Bursty and non-bursty neurons of both layers are shown in different colors. B, Proportion of bursty and non-bursty neurons in layers II and III of the MEC. C, Mean spike-time autocorrelation ( \pm 12 or $+300 \mathrm{~ms}$ ) of bursty and non-bursty neurons. $\boldsymbol{D}$, Mean spike-time autocorrelation for bursty and non-bursty neurons in layers II and III. ${ }^{* * *} p<10^{-4}$.

tions of bursty and non-bursty neurons in layers II and III are shown in Figure 9D. Together, these findings suggest that the presence of bursty and non-bursty neurons in layers II and III is a common feature of the MEC in several mammalian species.

\section{Discussion}

In this study, we found that principal neurons of the superficial layers of the MEC can be classified into two groups according to their tendency to fire bursts of action potentials at $125-250 \mathrm{~Hz}$. Neurons firing bursts at this frequency were more likely to display grid cell spatial periodicity than non-bursty neurons. On the other hand, non-bursty neurons preferentially encoded head direction or border proximity. During theta oscillations, both cell groups fired at the end of the descending phase of theta cycles, but bursty neurons fired, on average, $6 \mathrm{~ms}$ earlier than non-bursty neurons. Compared with non-bursty neurons, bursty neurons had more excitatory interactions with putative interneurons and were the only ones to display interspike interval adaptation. These results demonstrate that the firing patterns at the millisec- 
ond time scale predict the type of spatial selectivity of MEC neurons and their connectivity in the network.

The bursty/non-bursty classification was based on a principal component analysis of the spike-time autocorrelations. MEC neurons were divided into two classes because examination of the first two principal components showed that neurons form a $\mathrm{C}$-shaped structure with two clear axes. Additional support for this classification came from the nearly inverse spike-time autocorrelation shapes in the two groups and from the clear bimodal distribution of MEC neurons after linear discriminant analysis. It should be mentioned that there was no discontinuity between bursty and non-bursty neurons. In fact, approximately $10 \%$ of the neurons appeared to be in an "intermediate" zone between the two classes. This suggests that bursty and non-bursty neurons are on a continuum where the extreme states are more likely than the intermediate states. Thus, their distribution can still be considered continuous rather than categorical.

An important question concerns the anatomical location of bursty and non-bursty neurons. Initial reports that most grid cells are found in layer II, whereas head-direction cells are absent from layer II (Sargolini et al., 2006), could suggest that bursty and non-bursty neurons are located in layers II and III, respectively. However, we favor a scenario in which bursty and non-bursty neurons are intermingled in layers II and III for the following reasons. First, recordings of anatomically identified neurons indicate that head-direction cells, like grid cells, are also present in layer II of the MEC (Tang et al., 2014). Second, our histological analysis showed that as we advanced the tetrodes on each day, the recording sites became progressively more superficial. Despite this, there was no change in the ratio of the numbers of bursty and non-bursty neurons recorded when comparing the first and last three recording days. Finally, the analysis of data recorded in rats also indicates that layer II of the MEC contains bursty and nonbursty neurons (Mizuseki et al., 2009). Based on these findings, we argue that bursty and non-bursty neurons are not strictly segregated across the superficial layers of the MEC. Interestingly, we found that the functional differences observed between bursty and non-bursty neurons were still present when considering only neurons recorded simultaneously on a tetrode with at least one neuron of the other class. This suggests that bursty and nonbursty neurons located $<100 \mu \mathrm{m}$ apart (Henze et al., 2000) are functionally distinct.

We found that putative excitatory monosynaptic connections detected via spike transmission probability originated predominantly from bursty neurons. Previous studies have established that these connections preferentially target parvalbumin-expressing interneurons (Royer et al., 2012; Buetfering et al., 2014). Thus, our data suggest that bursty neurons have a more direct influence on action potential generation in parvalbumin-expressing interneurons. Since stellate cells in layer II are synaptically connected to adjacent fastspiking interneurons with a probability approaching 0.6 (Pastoll et al., 2013), it is tempting to speculate that a majority of stellate cells have the firing pattern of bursty neurons. Interestingly, stellate cells in vitro fire bursts of action potentials upon strong depolarization and show pronounced interspike interval adaptation during spike bursts (Alonso and Klink, 1993). Also, when a classifier to identify putative stellate and pyramidal cells was applied to our data set (Tang et al., 2014), more bursty neurons were classified as putative stellate cells than pyramidal cells. It should be mentioned, however, that non-bursty neurons were as likely to be classified as putative stellate cells as to be classified as putative pyramidal cells. This suggests that the bursty/non-bursty classification captures functional features that are not well dissociated by the current stellate/pyramidal cell classi- fier. Ultimately, juxtacellular or whole-cell recordings of anatomically identified neurons will be needed to firmly establish the anatomical properties of bursty and non-bursty neurons described in this study. The shorter recording durations commonly reported with these two techniques should not be a major concern as we showed that highly accurate classification of bursty and non-bursty neurons is possible with recording sessions shorter than $10 \mathrm{~min}$. Since bursty and non-bursty neurons have distinct spike waveforms and excitatory interactions with interneurons, these two classes are likely to show important anatomical differences.

Grid cells have been suggested to play an important role in path integration, a process that allows an animal to update its estimate of its current position from internal cues generated during locomotion (Hafting et al., 2005; McNaughton et al., 2006). Attractor network models of grid cells are based on either direct or indirect recurrent connectivity between grid cells, where connection strength varies as a function of difference in grid phase (McNaughton et al., 2006; Burak and Fiete, 2009; Bonnevie et al., 2013; Pastoll et al., 2013). Updating the active position in the network depends on inputs from head-direction cells and additional speed-modulated inputs. When geometric information about the external world is available, the anchoring of grid cells to the environment could depend on the activity of border cells (Solstad et al., 2008). In the present study, we found that bursty and non-bursty neurons preferentially perform distinct functions in this network. Bursty neurons conveyed more spatial information, were more likely to express grid periodicity, and showed weaker head-direction selectivity, three properties that make them especially well suited to convey information about the current location of the animal. On the other hand, non-bursty neurons with lower spatial information and strong headdirection selectivity could encode the angular information required to update the representation of the current position when the animal moves. Finally, the activity of non-bursty border cells might provide an important source of information to anchor the grid representation to the external environment (Hardcastle et al., 2015).

\section{References}

Alonso A, Klink R (1993) Differential electroresponsiveness of stellate and pyramidal-like cells of medial entorhinal cortex layer II. J Neurophysiol 70:128-143. Medline

Bannerman DM, Yee BK, Lemaire M, Wilbrecht L, Jarrard L, Iversen SD, Rawlins JN, Good MA (2001) The role of the entorhinal cortex in two forms of spatial learning and memory. Exp Brain Res 141:281-303. CrossRef Medline

Bjerknes TL, Moser EI, Moser MB (2014) Representation of geometric borders in the developing rat. Neuron 82:71-78. CrossRef Medline

Bonnevie T, Dunn B, Fyhn M, Hafting T, Derdikman D, Kubie JL, Roudi Y, Moser EI, Moser MB (2013) Grid cells require excitatory drive from the hippocampus. Nat Neurosci 16:309-317. CrossRef Medline

Brandon MP, Bogaard AR, Libby CP, Connerney MA, Gupta K, Hasselmo ME (2011) Reduction of theta rhythm dissociates grid cell spatial periodicity from directional tuning. Science 332:595-599. CrossRef Medline

Buetfering C, Allen K, Monyer H (2014) Parvalbumin interneurons provide grid cell-driven recurrent inhibition in the medial entorhinal cortex. Nat Neurosci 17:710-718. CrossRef Medline

Burak Y, Fiete IR (2009) Accurate path integration in continuous attractor network models of grid cells. PLoS Comput Biol 5:e1000291. CrossRef Medline

Burgalossi A, Herfst L, von Heimendahl M, Förste H, Haskic K, Schmidt M, Brecht M (2011) Microcircuits of functionally identified neurons in the rat medial entorhinal cortex. Neuron 70:773-786. CrossRef Medline

Csicsvari J, Hirase H, Czurko A, Buzsáki G (1998) Reliability and state dependence of pyramidal cell-interneuron synapses in the hippocampus: an ensemble approach in the behaving rat. Neuron 21:179-189. CrossRef Medline 
Csicsvari J, Hirase H, Czurkó A, Mamiya A, Buzsáki G (1999) Oscillatory coupling of hippocampal pyramidal cells and interneurons in the behaving rat. J Neurosci 19:274-287. Medline

Day M, Langston R, Morris RG (2003) Glutamate-receptor-mediated encoding and retrieval of paired-associate learning. Nature 424:205-209. CrossRef Medline

Domnisoru C, Kinkhabwala AA, Tank DW (2013) Membrane potential dynamics of grid cells. Nature 495:199-204. CrossRef Medline

Dupret D, O’Neill J, Csicsvari J (2013) Dynamic reconfiguration of hippocampal interneuron circuits during spatial learning. Neuron 78:166180. CrossRef Medline

Fyhn M, Hafting T, Witter MP, Moser EI, Moser MB (2008) Grid cells in mice. Hippocampus 18:1230-1238. CrossRef Medline

Hafting T, Fyhn M, Molden S, Moser MB, Moser EI (2005) Microstructure of a spatial map in the entorhinal cortex. Nature 436:801-806. CrossRef Medline

Hafting T, Fyhn M, Bonnevie T, Moser MB, Moser EI (2008) Hippocampusindependent phase precession in entorhinal grid cells. Nature 453:1248-1252. CrossRef Medline

Hardcastle K, Ganguli S, Giocomo LM (2015) Environmental boundaries as an error correction mechanism for grid cells. Neuron 86:1-13. CrossRef Medline

Hargreaves EL, Rao G, Lee I, Knierim JJ (2005) Major dissociation between medial and lateral entorhinal input to dorsal hippocampus. Science 308: 1792-1794. CrossRef Medline

Henze DA, Borhegyi Z, Csicsvari J, Mamiya A, Harris KD, Buzsáki G (2000) Intracellular features predicted by extracellular recordings in the hippocampus in vivo. J Neurophysiol 84:390-400. Medline

Heys JG, Rangarajan KV, Dombeck DA (2014) The functional microorganization of grid cells revealed by cellular-resolution imaging. Neuron 84:1079-1090. CrossRef Medline

Huxter J, Burgess N, O'Keefe J (2003) Independent rate and temporal coding in hippocampal pyramidal cells. Nature 425:828-832. CrossRef Medline

Huxter JR, Senior TJ, Allen K, Csicsvari J (2008) Theta phase-specific codes for two-dimensional position, trajectory and heading in the hippocampus. Nat Neurosci 11:587-594. CrossRef Medline

MacDonald CJ, Lepage KQ, Eden UT, Eichenbaum H (2011) Hippocampal "time cells" bridge the gap in memory for discontiguous events. Neuron 71:737-749. CrossRef Medline

Marshall L, Henze DA, Hirase H, Leinekugel X, Dragoi G, Buzsáki G (2002) Hippocampal pyramidal cell-interneuron spike transmission is frequency dependent and responsible for place modulation of interneuron discharge. J Neurosci 22:RC197(1-5). Medline

Maurer AP, Cowen SL, Burke SN, Barnes CA, McNaughton BL (2006) Phase precession in hippocampal interneurons showing strong functional coupling to individual pyramidal cells. J Neurosci 26:13485-13492. CrossRef Medline

McNaughton BL, Battaglia FP, Jensen O, Moser EI, Moser MB (2006) Path integration and the neural basis of the "cognitive map." Nat Rev Neurosci 7:663-678. CrossRef Medline
Mizuseki K, Sirota A, Pastalkova E, Buzsáki G (2009) Theta oscillations provide temporal windows for local circuit computation in the entorhinalhippocampal loop. Neuron 64:267-280. CrossRef Medline

Mizuseki K, Sirota A, Pastalkova E, Diba K, Buzsáki G (2013) Multiple single unit recordings from different rat hippocampal and entorhinal regions while the animals were performing multiple behavioral tasks. Collaborative Research in Computational Neuroscience-data sharing, http://crcns. org/data-sets/hc/hc-3. CrossRef

Morris RG, Garrud P, Rawlins JN, O'Keefe J (1982) Place navigation impaired in rats with hippocampal lesions. Nature 297:681-683. CrossRef Medline

Pastoll H, Solanka L, van Rossum MC, Nolan MF (2013) Feedback inhibition enables theta-nested gamma oscillations and grid firing fields. Neuron 77:141-154. CrossRef Medline

Ray S, Naumann R, Burgalossi A, Tang Q, Schmidt H, Brecht M (2014) Grid-layout and theta-modulation of layer 2 pyramidal neurons in medial entorhinal cortex. Science 343:891-896. CrossRef Medline

Royer S, Zemelman BV, Losonczy A, Kim J, Chance F, Magee JC, Buzsáki G (2012) Control of timing, rate and bursts of hippocampal place cells by dendritic and somatic inhibition. Nat Neurosci 15:769-775. CrossRef Medline

Sargolini F, Fyhn M, Hafting T, McNaughton BL, Witter MP, Moser MB, Moser EI (2006) Conjunctive representation of position, direction, and velocity in entorhinal cortex. Science 312:758-762. CrossRef Medline

Savelli F, Yoganarasimha D, Knierim JJ (2008) Influence of boundary removal on the spatial representations of the medial entorhinal cortex. Hippocampus 18:1270-1282. CrossRef Medline

Schmidt-Hieber C, Häusser M (2013) Cellular mechanisms of spatial navigation in the medial entorhinal cortex. Nat Neurosci 16:325-331. CrossRef Medline

Schmitzer-Torbert N, Jackson J, Henze D, Harris K, Redish AD (2005) Quantitative measures of cluster quality for use in extracellular recordings. Neuroscience 131:1-11. CrossRef Medline

Skaggs WE, McNaughton BL, Wilson MA, Barnes CA (1996) Theta phase precession in hippocampal neuronal populations and the compression of temporal sequences. Hippocampus 6:149-172. CrossRef Medline

Solstad T, Boccara CN, Kropff E, Moser MB, Moser EI (2008) Representation of geometric borders in the entorhinal cortex. Science 322:18651868. CrossRef Medline

Steffenach HA, Witter M, Moser MB, Moser EI (2005) Spatial memory in the rat requires the dorsolateral band of the entorhinal cortex. Neuron 45:301-313. CrossRef Medline

Tang Q, Burgalossi A, Ebbesen CL, Ray S, Naumann R, Schmidt H, Spicher D, Brecht M (2014) Pyramidal and stellate cell specificity of grid and border representations in layer 2 of medial entorhinal cortex. Neuron 84: 1191-1197. CrossRef Medline

Varga C, Lee SY, Soltesz I (2010) Target-selective GABAergic control of entorhinal cortex output. Nat Neurosci 13:822-824. CrossRef Medline

Zhang SJ, Ye J, Miao C, Tsao A, Cerniauskas I, Ledergerber D, Moser MB, Moser EI (2013) Optogenetic dissection of entorhinal-hippocampal functional connectivity. Science 340:1232627. CrossRef Medline 